\title{
Cancer coopts differentiation of B-cell precursors into macrophages
}

\section{Chen Chen}

National Institute on Aging

\section{Bongsoo Park}

National Institute on Aging

\section{Emeline Ragonnaud}

National Institute on Aging

\section{Monica Bodogai}

National Institute on Aging

\section{Le Zong}

$\mathrm{NIA} / \mathrm{NIH}$

\section{Jung-Min Lee}

Center for Cancer Research

\section{Isabel Beerman}

National Institute on Aging

Arya Biragyn ( $\nabla$ biragyna@mail.nih.gov )

National Institute on Aging https://orcid.org/0000-0001-5276-6102

\section{Article}

Keywords: B-cell precursors, macrophage, transdifferentiation, immune suppression, RNA profiling

Posted Date: September 13th, 2021

DOl: https://doi.org/10.21203/rs.3.rs-858433/v1

License: (c) (1) This work is licensed under a Creative Commons Attribution 4.0 International License. Read Full License

Version of Record: A version of this preprint was published at Nature Communications on September 14th, 2022. See the published version at https://doi.org/10.1038/s41467-022-33117-y. 


\section{Cancer coopts differentiation of B-cell precursors into macrophages}

Chen Chen ${ }^{1}$, Bongsoo Park ${ }^{2}$, Emeline Ragonnaud ${ }^{1}$, Monica Bodogai ${ }^{1}$, Le Zong ${ }^{2}$, Jung-Min Lee $^{3}$, Isabel Beerman ${ }^{2 *}$ and Arya Biragyn ${ }^{1,4,5^{*}}$

From: ${ }^{1}$ Immunoregulation Section, Laboratory of Immunology and Molecular Biology;

${ }^{2}$ Epigenetics and Stem Cell Aging Unit, Translational Gerontology Branch, and National Institute on Aging, Baltimore, MD; ${ }^{3}$ Women's Malignancies Branch, Center for Cancer Research, National Cancer Institute, Bethesda, MD ; ${ }^{4}$ Lead contact; ${ }^{5}$ Senior author

* Corresponding authors: Arya Biragyn, Ph.D., E-mail: biragyna@mail.nih.gov and Isabel Beerman, Ph.D., E-mail: isabel.beerman@nih.gov

Running title: B cells transdifferentiation in cancer

Keywords: B-cell precursors, macrophage, transdifferentiation, immune suppression, RNA profiling

Conflict-of-interest disclosure: The authors declare no conflicting financial interests 


\begin{abstract}
We recently reported that some cancers induce accumulation of bone marrow (BM) B-cell precursors in the spleen to convert them into metastasis-promoting, immunosuppressive B cells. Here, using various murine tumor models and samples from humans with breast and ovarian cancers, we provide evidence that cancer cells also coopt differentiation of the extra nodal B-cell precursors to generate macrophages (termed B-MF). We link the transdifferentiation to a small subset of CSF $1 \mathrm{R}^{+}$Pax $5^{\text {Low }}$ cells within BM pre-B and immature B cells and cancer-secreted M-CSF that downregulates Pax5 via CSF1R signaling. Thus, cancer generates tumor-associated macrophages (TAM) from B-cell precursors in addition to their primary source, monocytes. Based on their differences from monocyte-derived TAM, such as a superior ability to induce FoxP3 ${ }^{+}$Tregs, suppress proliferation of $\mathrm{T}$ cells and more efficiently phagocytize apoptotic cells, we propose that cancer generates B-MF to mediate cancer escape.
\end{abstract}


The role of B cells in cancer remains poorly understood despite reports of both positive and negative associations of B cells with the disease outcome. Even in the same murine model, T2-MZ B cells and aging-associated B cells can either increase or mitigate B16-F10 melanoma growth in C57BL/6 mice, respectively $(1,2)$. B cells can also inhibit or enhance metastasis in BALB/c mice with orthotopic $4 \mathrm{~T} 1$ breast cancer cells $(3,4)$. At least some cancer-promoting functions of $\mathrm{B}$ cells are attributed to their immunosuppressive, regulatory subsets termed Bregs, whose generation and function appear to be actively controlled by cancer itself. To enforce this regulation, cancer cells secrete various factors that affect survival and differentiation of B cells, including B lymphocyte stimulator (BLyS/BAFF), thymic stromal lymphopoietin (TSLP), colony stimulating factors (CSF such as M-CSF, GM-CSF and G-CSF) and lipid mediators such as 5-lipoxygenase (5-LO) metabolites (5-10). In BALB/c mice with orthotopic 4T1 breast cancer, a model for human triple negative breast cancer (11), our group linked 5-LO metabolite-induced activation of PPAR $\alpha$ to the conversion of B cells into $\operatorname{TGF} \beta^{+} \mathrm{CD} 25^{+}$ Bregs (tBregs) (7). These converted cells then promote metastasis acting as potent suppressors of antitumor $\mathrm{T}$ cell activity and inducers of FoxP3 ${ }^{+}$Tregs and MDSCs via the TGF $\beta /$ TGF $\beta$ RII axis $(5,12)$. Breast and ovarian cancer cells secret TSLP both to prepare the metastasis "soil" in the lungs and to accumulate BM B-cell precursors in the spleen for conversion to tBregs. In the lungs, TSLP enables infiltration of CCR $4^{+}$cancer cells and their protector CCR $4^{+} \mathrm{FoxP}^{+}$Tregs by upregulating expression of $\operatorname{CCL} 17(6,13)$, while in the BM, TSLP downregulates expression of CXCR4 and $\alpha 4 \beta 1$ in B-cell precursors, causing their premature emigration and accumulation in the spleen to be converted into $\mathrm{TGF}^{+} \mathrm{CD} 25^{+}$tBregs (10). M-CSF and GM-CSF from cancer cells promote differentiation 
and survival of cancer-promoting myeloid suppressive cells and tumor-associated macrophages (TAM) from BM monocytes $(8,9)$. Although bifurcation of myeloid and lymphoid lineage from multipotent progenitors occurs before specialization of B-cell progenitors in BM, surprisingly some B-cell precursors appear to retain macrophage differentiation potential. For example, the differentiation of BM B-cell precursors can be diverted towards macrophages upon forced expression or deletion of a single transcription factor $(14,15)$. Anecdotal reports indicate that this transdifferentiation can also occur in $\mathrm{BM}$ of naïve mice, which is linked to a small subset of biphenotypic $\left(\mathrm{CD} 19^{+} \mathrm{B} 220^{+}\right.$

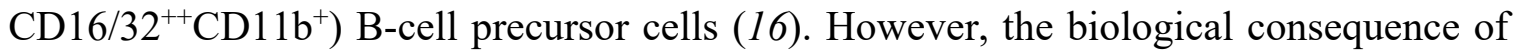
this rare event and whether cancers affect this process remain unknown.

Here, we report that cancers frequently induce the transdifferentiation of the bona fide $\mathrm{BM}$ Csf1 $\mathrm{R}^{+} \mathrm{Pax} 5^{\text {Low }}$ pre-B and immature B (iB) cells into TAM (termed B-MF). To do this, cancers first drive accumulation of B-cell precursors/iB cells in the spleen and tumors. Unlike "classical" monocyte-derived TAM (9), cancer appears to use B-MF to mediate cancer escape via suppression of antitumor T cells. This does not appear to be a mouse specific phenomenon, as B-MF can be detected in patients with breast and ovarian cancers, and we present evidence of B-MF signatures in published scRNA sequence data of human cancers.

\section{RESULTS}

B-cell markers are expressed in TAM. We previously reported that some cancers mobilize BM B-cell precursors in the spleen and tumors (10) to subsequently convert them into TGF $\beta^{+}$tBregs $(5,7,12)$. Microarray transcription profiling of B-cell precursors and 
tBregs from mice with BALB/c mice with orthotopic $4 \mathrm{~T} 1.2$ breast cancer surprisingly revealed significant upregulation of macrophage-associated genes, such as CD68, Csflr (encodes CSF1R), Cebpb (CCAAT Enhancer binding protein beta), Cebpg (CCAAT Enhancer binding protein gamma), $C c l 2$ (CCL2), and $C s f 1$ (M-CSF) (Fig. S1A). Given that CEBPB and CSF1R play essential roles in defining macrophage fate $(14,17)$ and anecdotal reports on retention of macrophage differentiation potential in B-cell precursors $(16,18)$, we wondered whether the upregulation of macrophage genes indicates that cancer hijacks B-cell differentiation, pushing them to macrophages. To test this possibility, we FACS evaluated tumor-infiltrating B cells (TIB, gated on $\mathrm{CD} 19^{+}$) and TAM (gated on $\mathrm{F} 4 / 80^{+}$) in WT BALB/c mice and $\mu \mathrm{MT}$ BALB/c mice (B-cell differentiation halted at the pro-B-cell stage (19)) bearing orthotopic 4T1.2 breast cancer. While both TIB and TAM in WT mice contained a small but significant proportion of cells co-expressing B-cell and macrophage markers, the double lineage expressing cells were not present in $\mu \mathrm{MT}$ mice (Fig. 1A, gating strategy is in Fig. S1B). We also evaluated if these cells were present in other tumor models. TIB and TAM co-expressing B-cell and macrophage markers were found in WT BALB/c mice with orthotopic EMT6 breast cancer, and WT C57BL/6 mice with other orthotopic cancers (AT3 breast and MC38 colon cancer) or spontaneous ovarian cancer (Mogp), but were not detected in $\mu \mathrm{MT}$ or $\mathrm{J}_{\mathrm{H}} \mathrm{T}$ mice with these cancers (Fig. 1B and Fig. $\mathrm{S} 1 \mathrm{C}, \mathrm{D})$. Of note, in $\mathrm{J}_{\mathrm{H}} \mathrm{T}$ mice, B-cell differentiation is blocked at the pro-B-cell stage (20). A comprehensive FACS evaluation of TAM revealed they do indeed express additional Bcell-specific markers, including Mb1/CD79a, CD20, IgM, and IgD (Fig. S1D-G). To further confirm our finding, we used a B-cell lineage tracer Mb1-EYFP mouse model (21), which expresses enhanced yellow fluorescent protein (EYFP) as a readout for the Ig- $\alpha$ 
receptor exclusively expressed in B cells (except plasma cells). Upon challenge with MC38 tumor cells, $\mathrm{EYFP}^{+} \mathrm{F} 4 / 80^{+} \mathrm{CD} 11 \mathrm{~b}^{\text {inter/low }} \mathrm{TAM}$ were significantly increased in the tumor (Fig. S2). In sum, cancers cause a marked increase of macrophage-like cells derived from B cells (hereafter referred to as B-MF).

Cancer induces B-cell-to-macrophage differentiation. To explain the accumulation of B-MF, we tested whether cancer cells trans-differentiate them from B cells. Sort-purified $\mathrm{Lin}^{-} \mathrm{CD} 19^{+} \mathrm{B} 220^{+} \mathrm{B}$ cells ( $>98 \%$ purity) from naïve mouse bone marrow (BM), spleen, lymph nodes (LN) and peritoneal cavity (PeC) were cultured for 7 days in conditioned medium (CM) of 4T1.2 cancer cells (4T1.2-CM). Of note, unless specified, from here on this incubation time was chosen because of a noticeable upregulation of macrophage markers (Fig. S3A). 4T1.2-CM, but not control medium, significantly upregulated F4/80 and CD11b in B cells from BM, but not from spleen, $\mathrm{LN}$ or PeC (Fig. 1C). The BM B cells acquired features associated with macrophages, such as adherence to plastic, enlarged vacuolar cytoplasm, and ability to phagocytize fluorochrome-labelled $E$. coli (Fig. 1D and Fig. S3B). Independent experiments with sort-purified B-cell subsets revealed that only BM B-cell precursors and iB cells, but not splenic transitional, follicular (FOB), or marginal zone (MZB) B cells, can become B-MF (Fig. S3C; gating strategy in Fig. S3D). Consistent with the accumulation of B-MF in mice with various tumors, CM from almost every type of cancer cell (except B16-F10 melanoma cells) induced the generation of B-MF from BM B-cell precursors/iB cells (BMBP) and pre-B-cell line 70z/3 (Fig. 1E-G and Fig. S3E). In single cell Imagestream FACS analysis, macrophage and Bcell markers were indeed co-expressed on single B-MF cells derived from BM Mb1-EYFP ${ }^{+}$ B cells (Fig. 1H). To rule out artifacts of in vitro experiments, we also performed a 7-day 
in vivo conversion assay by i.p. implanting sort-purified BMBP from naïve Mb1-EYFP mice into PeC of C57BL/6 mice with or without ID8 ovarian cancer in PeC. Tumor-bearing mice contained significantly higher frequency of $\mathrm{EYFP}^{+} \mathrm{B}-\mathrm{MF}$ in $\mathrm{PeC}$ than naïve mice (Fig. 1I and Fig. S3F). These cells also markedly upregulated TGFß/LAP and PD-L1 (Fig. 1I), the two factors utilized in immunoregulation $(22,23)$. Taken together, we concluded that cancer uses BMBP to generate TAM in addition to their hitherto known source, monocytes (9).

B-MF and Mo-MF differ functionally. To understand why cancer generates TAM from B cells, we ex vivo generated B-MF and monocyte-derived macrophages (Mo-MF) from sort-purified BMBP and monocytes, respectively, by stimulating with 4T1.2-CM, as described above. While both populations adhered to plastic and comparably upregulated F4/80 and CD11b, only B-MF expressed the B-cell-specific markers (CD79a and IgM, Fig. 2A, B and Fig. S4A). mRNA microarray analysis of B-MF and Mo-MF revealed that regardless of origin, the two populations share expression of numerous macrophage-related genes and do not robustly expresses B-cell signature profiles (Fig. S4B-D and Table S1). Principal component analysis (PCA) and transcription profiling clearly separate B-MF from Mo-MF (Fig. 2C and Table S2). B-MF expressed higher levels of genes involved in fatty acid metabolism, cell cycle, steroid-cholesterol biosynthesis and downregulated expression of pro-inflammatory and IFN $\gamma$ response genes (Fig. 2D, E). While Mo-MF had more robust M1-like transcription, M2-skewing was more pronounced in B-MF (Fig. 2E and Fig. S4E). The unique transcription profiles were also confirmed in single cell RNA sequencing (scRNA-seq) of in vitro generated B-MF $(10,563)$ and Mo-MF $(10,235)$ cells, with UMAP clustering identifying mostly separate cell-clusters of macrophages derived 
from either B cells or monocytes (Fig. 2F and Table S3). We distinguished 12 cell clusters with the Leiden algorithm, using shared nearest neighbor (SNN) in PCA space and identified the key genes establishing the six clusters accounting for the majority of single cells (Fig. 2G, H and Fig. S4F). B-MF appeared to be more phagocytic than Mo-MF, as they markedly upregulated Mrcl (encodes CD206, Fig. 2I). Given the unique transcriptional signatures of the B-MF, we next examined scRNA profiles from four independent samples of TAM in mice with 4T1.2 cancer (Fig. 2J). Using signature genes identified from in vitro generated macrophages (Fig. $2 \mathrm{H})$ we noted three clusters $(0,6$, and 8) with robust expression of genes identified in B-MF (Fig. S4H). Cluster 8 also strongly overlapped with a mixed macrophage population (Cluster 4, Fig. 2G and Fig. S4F-H), suggesting that only clusters 0 and 6 were most similar to B-MF. We also examined expression of three genes robustly expressed in B-MF and they were also expressed in TAM clusters 0 and 6 (Fig. 2K). In contrast, expression of key Mo-MF genes was mostly found in clusters 1, 3, 4, and 5 (Fig. S4G), suggesting B-MF and Mo-MF retain traceable and different transcription profiles in vivo.

To confirm B-MF and Mo-MF differences at the functional level, first we examined their proliferation. Only B-MF expressed higher levels of Ki67 and readily incorporated BrdU (pulsed at day 6 and tested at day 7 of the culture) (Fig. 3A, B and Fig. S5A), indicating their proliferative and self-maintaining state. Given the changes seen in fatty acid metabolism (Fig. 2D), we stained the cells with filipin III, a fluorescent polyene antibiotic that binds to free cholesterol (24). Compared with Mo-MF, the cellular cholesterol content was significantly upregulated in both in vitro and in vivo generated BMF (Fig. 3C, D and Fig. S1E-G). To evaluate phagocytosis, B-MF and Mo-MF were 
cultured with fluorochrome-labeled apoptotic cancer cells. B-MF phagocytized apoptotic cells significantly better than Mo-MF (Fig. 3E, F). Given B-MF markedly upregulated TGF $\beta /$ LAP and PD-L1 (Fig. 1I), we tested their function in a 4-day T-cell suppression assay (5). When B-MF or Mo-MF were cultured with naïve mouse splenic $\mathrm{T}$ cells stimulated with anti-CD3/CD28 antibodies, only B-MF significantly inhibited proliferation of $\mathrm{CD}^{+} \mathrm{T}$ cells and, at lesser extent, $\mathrm{CD} 8^{+} \mathrm{T}$ cells (Fig. 3G and Fig. S5B-E). Because TGF $\beta$ is involved in induction of FoxP $3^{+}$Tregs (5), we also performed an in vitro Treg conversion assay by culturing the two macrophages with splenic $\mathrm{CD} 25^{-} \mathrm{CD} 4^{+} \mathrm{T}$ cells in the presence of anti-CD3/CD28 antibodies and IL2 for 4 days. Compared with Mo-MF, B-MF induced higher levels of FoxP3 ${ }^{+}$Tregs (Fig. S5F), further suggesting that cancer presumably uses B-MF to mediate immunoregulation and thereby tumor progression. We therefore tested whether B-MF can reverse the retarded growth of B16-F10 melanoma in $\mu \mathrm{MT}$ mice (n=5/group, Fig. 3G), which we previously linked to the lack of B cells (12). Intravenous transfer of B-MF significantly decreased tumor-infiltrated $\mathrm{CD}^{+} \mathrm{T}$ cells (and at lesser extent $\mathrm{CD}^{+} \mathrm{T}$ cells) and their IFN-expressing subsets (Fig. 3H, I and Fig. S5H) and markedly increased growth of B16-F10 melanoma in $\mu \mathrm{MT}$ mice $(\mathrm{p}<0.05$, Fig. $3 \mathrm{~J}$ and Fig. S5G). In contrast, T cells in the spleen and dLN were not affected by the B-MF transfer (not depicted), suggesting that B-MF primarily regulate tumor-infiltrating $\mathrm{T}$ cells.

Cancer mobilizes BMBP in the spleen to convert them to B-MF. Recently, we reported that breast cancer induces tBregs from BM pre-B cells. To do this, cancer first stimulates accumulation of BMBP in the spleen and tumor by causing their premature emigration from BM (10). We therefore reasoned that cancer targets the same pool of BMBP accumulated in the spleen to generate B-MF. In support of this hypothesis, the total 
number of $\mathrm{CD} 93^{+} \mathrm{BMBP}$ were markedly increased in the circulation (but decreased in $\mathrm{BM}$ ) of mice with 4T1.2 and Mogp cancers (Fig. 4A and Fig. S6A, B) as compared with naïve mice (Fig. S6A, B). Stimulation with 4T1.2-CM readily generated B-MF from CD93+ BMBP sort-purified from spleens of mice with 4T1.2 cancer (Fig. S6C) but failed to do so if B cells were isolated from spleens of naïve mice, regardless of CD93 expression (Fig. S6C and Fig. S3C), implying that cancer first drives accumulation of BMBP in the spleen to convert them into B-MF.

Cancer targets CSF1 ${ }^{+} \mathrm{CD}^{+}{ }^{+}$BMBP by secreting M-CSF. To understand the mechanism of the B-MF generation, we analyzed $\mathrm{CM}$ of cancer cells for secreted factors that could affect differentiation of macrophages. M-CSF, a regulator of macrophage differentiation and survival $(8,17)$, was among the factors that was highly increased in the cancer cells that induce B-MF (Fig. 4B). Conversely, M-CSF was almost absent in CM from B16-F10 cells (Fig. 4B and Fig. S6D, E), which did not induce the generation of BMF (Fig. 1E and Fig. S3E). Compared to naïve mice, serum M-CSF was also significantly upregulated in mice with 4T1.2 cancer (Fig. S6F). Importantly, BM CD93 ${ }^{+} \mathrm{CD} 19^{+} \mathrm{BMBP}$ of naïve mice expressed its cognate receptor CSF1R (about $15 \%$ iB cells, $6 \%$ of pre-B cells and $1.5 \%$ pro-B cells, Fig. S6G). In mice with $4 \mathrm{~T} 1$ cancer, $\mathrm{CSF} 1 \mathrm{R}^{+} \mathrm{CD} 93^{+} \mathrm{CD} 19^{+} \mathrm{BMBP}$ were markedly reduced in BM but increased in the spleen (Fig. 4A and Fig. S6G), consistent with their cancer-induced emigration from BM, as discussed above. To link these $\mathrm{CSF} 1 \mathrm{R}^{+} \mathrm{CD} 93^{+} \mathrm{CD} 19^{+} \mathrm{BMBP}$ to the generation of $\mathrm{B}-\mathrm{MF}$, we performed the in vitro macrophage conversion assay by stimulating sort-purified CSF1R ${ }^{+}$and CSF1R- B cells from BM and spleen of naïve mice with 4T1.2-CM. While CSF1R ${ }^{+}$BM B cells readily generated B-MF, the BM CSF1R- subset failed to do so (Fig. 4C). Consistent with an 
inability of splenic CD93 ${ }^{+}$B cells of naïve mice to generate B-MF (Fig. S6C and Fig. S3C), we failed to convert naïve mouse splenic B cells into B-MF regardless of CSF1R expression (Fig. 4C). We also cultured primary BM BMBP or 70z/3 cells with 4T1.2-CM in the presence or absence of neutralizing M-CSF antibody (Ab) or Ki20227, a specific inhibitor of c-Fms/CSF1R (25). Both cells failed to generate B-MF upon M-CSF neutralization or CSF1R signaling inhibition (Fig. 4D, E and Fig. S6H, I). To rule out artifacts of in vitro assay, we created mice with conditional CSF1R deficiency in B cells (Mb1-CSF1R ${ }^{\text {Flox/Flox }}$ mice, gating strategy in Fig. S7). Unlike WT littermates or monocytes from Mb1-CSF1R ${ }^{\text {Flox/Flox }}$ mice, the loss of CSF1R in BMBP significantly impaired the cancer CM-induced B-MF differentiation (Fig. 4F, G). Of note, the residual macrophage differentiation seen in Fig. 4F is presumably due to CSF1R expression preceding Mb1 expression and thus only pro-B cells and onward will have Csflr deletion.

Given that PAX5 is the key pro-B cell factor that represses Csflr and other myeloid lineage-specific genes $(15,26)$, we reasoned that cancer decreases levels of this transcription factor using M-CSF. FACS staining confirmed that Pax5 was markedly decreased in $\mathrm{BM} \mathrm{CD} 93^{+} \mathrm{BMBP}$, particularly in $\mathrm{CSF} 1 \mathrm{R}^{+}$but not $\mathrm{CSF} 1 \mathrm{R}^{-}$subsets, from mice with 4T1.2 or Mogp cancer (Fig. 5A and Fig. S8A, C). Importantly, Pax5 was also significantly decreased in BM CSF $1 \mathrm{R}^{+}$BMBP from naïve mice and 70Z/3 cells upon treatment with 4T1-CM or M-CSF (Fig. 5B, C and Fig. S8B). As Pax5 deficiency alone is sufficient to render pro-B cells susceptible to myeloid differentiation (15), we concluded that cancer uses M-CSF to reduce expression of Pax 5 in $\mathrm{CSF} 1 \mathrm{R}^{+} \mathrm{CD} 93^{+} \mathrm{BMBP}$ and thereby promote macrophage differentiation. 
To further understand the B-cell susceptibility towards macrophage conversion, we analyzed chromatin accessibility by performing ATAC-seq on $\mathrm{CSF}^{+} \mathrm{R}^{+}$and CSF1R ${ }^{-}$ BMBP isolated from both BM and spleen. PCA clustering showed the most robust differences in chromatin profiles were driven by the location of the BMBP (BM vs spleen) regardless of CSF1R expression, driving the PC2 axis (blue \& purple vs orange \& green, Fig. 5D). The chromatin landscapes of the CSF1 $\mathrm{R}^{+}$and CSF1 $\mathrm{R}^{-} \mathrm{BMBP}$ isolated from $\mathrm{BM}$ (orange and green, Fig. 5D) also significantly differed from each other, driving the PC3 axis. We then examined the differentially accessible regions (DARs) between $\mathrm{CSF}_{\mathrm{R}} \mathrm{R}^{+}$and CSF1R- cells isolated from spleen or BM. Whereas comparisons between CSF1 ${ }^{+}$and CSF1R- cells from spleen did not show any differences reaching our threshold for significance, confirming their close clustering on the $\mathrm{PC} 3$ axis; the $\mathrm{BM} \mathrm{CSF} 1 \mathrm{R}^{+}$cells contained significantly more open chromatin than the BM CSF1R-cells (Fig. 5D, E). These data suggest the BM CSF1 $\mathrm{R}^{+} \mathrm{BMBP}$ may have a more permissive chromatin environment, susceptible to macrophage differentiation signals. As the spleen-derived B cells and BM CSF1R- cells were refractory to macrophage conversion (Fig. 4C), we looked at DARs with less accessibility in CSF1R- compared to CSF1 $\mathrm{R}^{+} \mathrm{BM}$ cells (749 loci) in spleen cells to determine if these regions remain closed and potentially "lock in" the lymphoid lineage potential. Indeed, the overwhelming majority of regions with decreased accessibility in the BM CSF1R ${ }^{-}$cells remained closed in the cells from spleen (679 of 749). Evaluation of these consensus open regions found in $\mathrm{BM} \mathrm{CSF} 1 \mathrm{R}^{+}$cells for potential transcription factor binding sites permitting macrophage differentiation showed significantly increased accessibility of ERG and RUNX1 sites (Fig. 5F). ERG is known to be expressed both in myeloid and lymphoid progenitor cells (27) and has particular importance in early 
hematopoietic progenitor cells as it binds to coregulators such as RUNX and GATA (28). RUNX1 regulates growth and survival of macrophages via binding to promoter and enhancer regions of Csflr and upregulating its expression (29). Runx1 is also robustly expressed in early progenitor and myeloid-committed progenitor cells (30). Thus, the increased accessibility to binding sites of both ERG and RUNX1 suggests a potentially more primitive, permissive chromatin state allowing for myeloid lineage transformation of the BM CSF1 $\mathrm{R}^{+}$cells.

B-MF-generating CSF1 ${ }^{+}$BMBP accumulate in humans with cancer. We recently reported that peripheral mobilization of BMBP also occurs in humans with breast cancer (BC) (10), suggesting the generation of B-MF. To test this possibility, we FACS evaluated $\mathrm{PB}$ of healthy donors (HD, $\mathrm{n}=7)$ and patients with $\mathrm{BC}(\mathrm{n}=8)$. Compared with $\mathrm{HD}$, PB of $\mathrm{BC}$ was markedly increased in CSF1 $\mathrm{R}^{+} \mathrm{BMBP}$ (Fig. S8D), as we described in mice with cancer. Moreover, microarray transcription profiling of sort-purified B cells from $\mathrm{PB}$ of $\mathrm{BC}$ patients revealed that they significantly upregulated macrophage-associated genes, such as Cebpa, Marco, and Csf1r, as compared with B cells from HD (Fig. 5G). We also FACS evaluated B-cells from PB of patients with ovarian cancer $(\mathrm{OC}, \mathrm{n}=5)$. Compared with HD, OC patients significantly increased CSF1R ${ }^{+}$BMBP (Fig. 5H and Fig. S8E) with upregulated expression of CD68 and LDLR (Fig. 5H), similar to mice with cancer. Using recently published scRNA-seq data of tumor-infiltrated immune cells from patients with breast cancer (31), we also found a macrophage cluster with overlapping signatures of BMF-like cells (cluster 3, Fig. 5I) by examining genes with differential expression defined in murine in vitro-generated B-MF (cluster 0, Fig. 2H). In particular, cluster 3 was enriched for expression of EGR1, IER2, IER3, and SLC40A1, which were major drivers of identity 
for murine in vitro-generated B-MF (Fig. 5I). Similarly, in the single-cell transcriptome data from human high-grade serous OC (32), we also detected the B-MF-like signature in macrophages (Cluster 0, Fig. 5J), although with a lesser overlap than in $\mathrm{BC}$, further suggesting that human cancers can promote the B-cell transdifferentiation into macrophages.

\section{DISCUSSION}

BMBP undergo a series of subsequent and tightly regulated differentiation steps after their bifurcation from multipotent cells to committed myeloid/lymphoid lineage cells. Despite this, experiments with forced expression or inhibition of a single transcription factor or mutations that drive leukemogenesis $(14,15)$ reveal that BMBP retain plasticity and myeloid transdifferentiation potential. Unlike these artificial or rare events, here we report that the B-cell-to-macrophage transdifferentiation is commonly used by murine cancers to generate TAM/B-MF. In PB of humans with metastatic/recurrent triple-negative $\mathrm{BC}$ and high-grade serous $\mathrm{OC}$, we also detect a significant increase of CSF1R $\mathrm{CD}^{+} 8^{+} \mathrm{LDLR}^{+}$BMBP which also express the macrophage-specific genes Cebpa, Cebpb, and Marco. Importantly, the B-MF signature is also identifiable within unique macrophage clusters using recently published scRNAseq profiles of tumor-infiltrating cells in patients with BC (31) and high-grade serous OC (32). Although the characterization of human BMF is a topic of a separate study, our results suggest that human and murine cancers transdifferentiate BMBP into macrophages, adding one more feature to the heterogeneity and plasticity of TAM. The inflammatory and antitumor activities of TAM at the early stages of tumor can shift to proangiogenic and tumor-supporting M2-like phenotypes as 
tumor progresses (33), presumably when B-MF would be induced. Interestingly, B-MF resemble both small (S)-TAM and large (L)-TAM (which associate with a poor disease outcome) recently identified in human colorectal liver metastasis (34). The lipid metabolism and phagocytosis genes of B-MF (Fasn, Pltp, Acat1, Clqa, and Clqb) are upregulated in L-TAM, while LDLR, Hmgcr as well S100a8, Vcan, and Thbs1 are increased in S-TAM.

Although TAM are primarily derived from monocytes (9), our results clearly show that at least some originate from bona fide B cells. The biological relevance of this redundancy in generation of TAM remains poorly understood; however, based on our comparisons of the side-by-side generated B-MF and Mo-MF, we think that the two macrophages may serve different purposes. Transcriptionally, B-MF preferentially upregulate expression of genes involved in cell cycle, fatty acid metabolism, and steroidcholesterol biosynthesis, implying they utilize unique metabolic and inflammatory functions. Unlike Mo-MF, B-MF proliferate, i.e. self-maintain and thus may persist longer in the tumor. B-MF markedly upregulate surface expression of LDLR, which removes extracellular cholesterol/LDL (35), and this could explain the higher levels of intracellular cholesterol and lipids in B-MF compared to Mo-MF. Consistent with significant upregulation of genes associated with phagocytosis, M2-skewing and immunosuppressive functions (PD-L2, B7-H3, Marco, TGF $\beta$ ) and downregulation of pro-inflammatory and IFN $\gamma$ response genes, B-MF express higher levels of surface MRC1 (CD206), PD-L1 (CD274), and TGF $\beta /$ LAP and efficiently phagocytize apoptotic cells compared to Mo-MF. This efficient phagocytosis presumably occurs without overt inflammation, as LDLRmediated cholesterol influx inhibits activation of the inflammasome (36). Our data show 
that cancer generates phenotypically and functionally non-redundant TAM from BMBP and monocytes, where B-MF appear to promote cancer growth presumably by controlling antitumor T cell responses. First, unlike Mo-MF, B-MF efficiently suppress proliferation of $\mathrm{T}$ cells or induce the generation FoxP3 ${ }^{+}$Tregs in vitro. Second, B-MF enhance growth of B16-F10 melanoma in $\mu \mathrm{MT}$ mice. To do this, they primarily decrease $\mathrm{CD}^{+} \mathrm{T}$ cells and inhibit IFN $\gamma^{+} \mathrm{CD}^{+} \mathrm{T}$ cells in the tumor, presumably by utilizing the B-MF-expressed immunoregulatory factors, TGF $\beta /$ LAP and PD-L1 $(22,23)$, and LDLR. For example, LDLR may enhance the TGF $\beta$ responsiveness of target T cells by removing extracellular LDL/cholesterol that impairs TGF $\beta$ binding and thus signaling via TGF $\beta$ RII/TGF $\beta$ R1 (37).

We propose that cancer primarily targets a small subset of $\mathrm{CSF} 1 \mathrm{R}^{+} \mathrm{Pax} 5^{\mathrm{Low}}$ pre-B cells and iB cells recently emigrated from BM. First, B-MF are not found in tumor-bearing mice with the B-cell differentiation blockage at the pro-B cell stage. Second, splenic transitional B cells from naïve mice do not generate B-MF regardless of their CSF1R expression state, as cancer first needs to mobilize BMBP into the circulation as the source of B-MF. We and others have reported that cancers use TSLP and G-CSF to mobilize BM pre-B cells and HSPS in the circulation $(10,38)$. We also find the chromatin accessibility landscape of $\mathrm{BM}$ CSF $1 \mathrm{R}^{+} \mathrm{BMBP}$ to be significantly more open and permissive to macrophage differentiation signals. In contrast, $\mathrm{CSF} 1 \mathrm{R}^{+}$and $\mathrm{CSF} 1 \mathrm{R}^{-}$splenic $\mathrm{B}$ cells and BM CSF1R- cells present a chromatin landscape that is refractory to macrophage conversion. The overwhelming majority of regions with decreased accessibility in the BM CSF1R' cells remained closed in the spleen (679 of 749), presumably "locking in" the state of lymphoid lineage potential. It appears that $\mathrm{BM} \mathrm{CSF} 1 \mathrm{R}^{+}$cells have a potentially more 
primitive, permissive chromatin state allowing for myeloid lineage transformation. The BM CSF1R ${ }^{+}$cells have more accessible ERG and RUNX1 binding sites, the two transcription factors expressed in myeloid and lymphoid progenitor cells (27) and early progenitor and myeloid-committed progenitor cells (30), respectively. Given that RUNX1 also upregulates expression of Csflr by binding to its promoter and enhancer regions (29) and that the PAX5 deletion alone removes the repression of Csflr and other myeloid lineage-specific genes and induces the BM B-cell precursor transdifferentiation $(15,26)$, we think that RUNX1 supports CSF1R expression in CSF1R ${ }^{+}$BMBP to downregulate Pax5 in response tonic and cancer-secreted M-CSF. Give that the CSF1 $\mathrm{R}^{+}$BMBP in our patients with metastatic/recurrent triple-negative $\mathrm{BC}$ and high-grade serous $\mathrm{OC}$ co-express macrophage-associated genes, human cancer may also target biphenotypic B-cell precursors reported to have a macrophage-differentiation potential (16). Overall, for the first-time we report the B-cell-to-macrophage transdifferentiation as a physiological and widely utilized phenomenon. Murine and possibly human cancers target the transdifferentiation to generate immunosuppressive TAM. 


\section{MATERIAL AND METHODS}

Mice, cell lines: The animal protocol was approved by the ACUC committee of the National Institute on Aging (ASP 322-LMBI-2022) under the Guide for the Care and Use of Laboratory Animals (NIH Publication No. 86-23, 1985). The study used young (8-12 weeks old) female mice bred and housed in the same, specific pathogen-free environment at the National Institute on Aging (NIA). C57BL/6J, BALB/CJ, R26R-EYFP (B6.129X1Gt (ROSA)26Sortm1(EYFP)Cos/J), Csf1r flox mice (B6.Cg-Csf1rtm1.2Jwp/J) and $\mu \mathrm{MT}$ mice (B6.129-Ighm-tm 1 Cgn/J) and $\mathrm{J}_{\mathrm{H}} \mathrm{T}$ mice $\left(\mathrm{J}_{\mathrm{H}} \mathrm{T} ; \mathrm{B} 6.129 \mathrm{P} 2-\operatorname{Igh}-J t m 1 C g n / \mathrm{J}\right)$ in C57BL/6 background mice were purchased from the Jackson Laboratory (Bar Harbor, ME); $\mu \mathrm{MT}$ mice in BALB/c background were a gift from Dr. Thomas Blankenstein (Max-DelbrückCenter for Molecular Medicine, Berlin, Germany) (39). Mb1-Cre mice in C57BL/6 background (B6.C(Cg)-Cd79atm1(cre) Reth/EhobJ) were a gift from Dr. Richard Maraia (National Institute of Child Health and Human Development, Bethesda, MD) (40). Mogptag (Mogp) mice in C57BL/6 background were a gift from professor Dr. I. Miyoshi (Tohoku University Graduate School of Medicine, Miyagi, Japan) (12). To create mice with B-cell specific EYFP reporter (Mb1-EYFP) or CSF1R deletion (Mb1-CSF1R ${ }^{\text {Flox/Flox }}$ ), Mb1-cre mice were bred with R26R-EYFP and Csflr ${ }^{\text {flox }}$ mice, respectively.

4T1.2 cells-were a gift from Dr. Robin L. Anderson (Peter McCallum Cancer Center, Melbourne, Australia); MC38 colonic adenocarcinoma cells were a gift from Dr. Jeffrey Schlom (National Cancer Institute, Bethesda, MD) (41); mammary carcinoma AT3 cells were a gift from professor Scott I. Abrams (Roswell Park Comprehensive Cancer Center, Buffalo, NY); ID8-p53--RFP (ID8) cells were gift from professor Sharon Stack (University of Notre Dame, IN); and EMT6 cells and melanoma B16-F10 cells were 
purchased from American Type Culture Collection (Manassas, VA). Cells were tested free of mycoplasma with Mycoplasma Detection Kits (Lonza Basel, Switzerland; and IDEXX BioAnalytics, Columbia, MO).

Tissues and blood processing: Human samples were collected with written informed consent at the Clinical Core Laboratory, NIA, under Human Subject Protocol \# 2003054 and Tissue Procurement Protocol \# 2003-071. Cancer patient PBMC were collected before the clinical trial (protocol \# NCT02203513, NCI) from people with metastatic/recurrent triple-negative breast cancer and high-grade serous ovarian cancer $(42,43)$. The trial was approved by the Institutional Review Board of the Center for Cancer Research, National Cancer Institute, Bethesda, MD, USA. All experiments were performed on PBMC which were cryopreserved after collection. Mouse BM cells were flushed out of femurs and tibias with cold cRPMI. Single cell suspension of BM, spleen, LN were prepared with $70 \mu \mathrm{m}$ strainer (Falcon, Bedford, MA). BM, spleen, and blood cells were treated with ACK buffer to remove red blood cells. Mouse tumor tissues were cut to $3-5 \mathrm{~mm}$ pieces and digested with mouse tumor dissociation kit (Miltenyi Biotec, Bergisch Gladbach, Germany) following the manufacturer's instruction.

Flow cytometry (FACS): For immune cell phenotyping, cells were pre-incubated with TruStain FcX $\mathrm{X}^{\mathrm{TM}}$ solution before immunostaining with different combinations of antimouse or anti-human Abs ( $1 \mu \mathrm{g}$ per $10^{6}$ cells, Suppl. Table 4$)$ and fixable viability dye, then fixed/permeabilized with eBioscience ${ }^{\mathrm{TM}}$ intracellular fixation \& permeabilization buffer (Thermo Fisher, Waltham, MA). The samples were evaluated on FACSymphonyTM (BD, Franklin Lakes, NJ), Amnis ImageStreamX MKII (Millipore, Burlington, MA) or 
CytoFLEX (Beckman Coulter, Brea, CA). The results were analyzed with FlowJo v10(BD), IDEAS (Millipore) or Cytoexpert 2.3 (Beckman).

Cancer CM media preparation and cytokine quantification: Cells were cultured in RPMI1640 or DMEM (for ID8 cells) supplemented with 10\% FBS, $1 \times$ HEPES, sodium pyruvate, nonessential amino acids solution, penicillin-streptomycin-glutamine (Gibco, Gaithersburg, MD), and $55 \mathrm{mmol} / \mathrm{L} \beta$-mercaptethanol in T75 flask to $70-80 \%$ confluency. $\mathrm{CM}$ was collected after $5 \mathrm{~min}$ centrifugation at $1500 \mathrm{rpm}$, filtered with $0.2 \mu \mathrm{m}$ filter, and stored at $-80^{\circ} \mathrm{C}$ as single use aliquots. For cytokine tests, confluent cells were cultured with RPMI without FBS for $24 \mathrm{~h}$. Mouse serum was collected using BD Microtainer ${ }^{\circledR}$ Tubes following the manufacturer's instruction. Cytokines and M-CSF in filtered CM or sera were evaluated with Quantikine ELISA kit (R\&D, Minneapolis, MN) or with Proteome Profiler Mouse XL Cytokine Array (R\&D). Images were captured and analyzed with Fiji software.

In vitro B-MF conversion: $\mathrm{BM} \operatorname{Lin}^{-}(\mathrm{TER} 119, \mathrm{CD} 11 \mathrm{~b}, \mathrm{Gr}-1, \mathrm{CD} 3 \varepsilon, \mathrm{NK} 1.1$ or CD49b, Ly6C, Ly6G, CD11c)- CD19+ B cells were isolated from C57BL/CJ or BALB/CJ mice using FACSAria ${ }^{\mathrm{TM}}$ Fusion sorter and $10^{6} / \mathrm{ml}$ B cells were cultured in $50 \%$ cancer CM in cRPMI for 7 days in Nunc ${ }^{\mathrm{TM}}$ Multidishes with UpCell ${ }^{\mathrm{TM}}$ Surface (Thermo Fisher) without changing media for 7 days. $70 \mathrm{z} / 3$ pre-B cells $\left(10^{5} / \mathrm{ml}\right)$ were cultured in $50 \%$ cancer $\mathrm{CM}$ for up to 30 days with replenishing culture medium every 3-4 days. Adherent cells (macrophages) were harvested by detaching them at $4^{\circ} \mathrm{C}$ for 15 mins in PBS. For Giemsa staining, B-MF were fixed with ethanol for 5 mins and Wright-Giemsa stained according to the manufacturer's instruction. CSF1R receptor signaling was blocked with Ki20227 (R\&D). 
In vitro assays: For bacterial uptake assay, E. coli (Thermo Fisher) labeled with pHrodo $^{\mathrm{TM}}$ red $(0.1 \mathrm{mg} / \mathrm{ml})$ were cultured with B-MF generated from RAG-GFP for $2 \mathrm{~h}$. Cells were washed with PBS, fixed with $4 \%$ formaldehyde and stained with DAPI. For phagocytosis of apoptotic cancer cells, ID8-RFP cells $\left(10^{6} / \mathrm{ml}\right)$ were pretreated with 300 nM gemcitabine hydrochloride (Sigma, St. Louis, MO) for 24h, then washed with PBS and cultured with macrophages for $2 \mathrm{~h}$. Macrophages were stained with anti-F4/80-FITC Ab and DAPI and phagocytosis was evaluated using Zeiss LSM 710 (Carl Zeiss AG, Jena, Germany) and analyzed with Fiji software. For macrophage proliferation test, BrdU (10 $\mu \mathrm{M}, \mathrm{BD})$ was added to macrophage cultures at day 5 and the BrdU incorporation was quantified at day 7 using FACSymphony ${ }^{\mathrm{TM}}$ and analyzed by FlowJo.

T cell suppression assay was described elsewhere (13). Briefly, splenic T cells isolated with $\mathrm{CD}^{+} \mathrm{T}$ cell enrichment column (R\&D) were labeled with eFluor ${ }^{\mathrm{TM}} 450$ and cultured with macrophages at 1:10, 1:20 and 1:40 E: T ratios in 96-well flat bottom plates coated with $5 \mu \mathrm{g} / \mathrm{ml}$ anti-mouse CD3e antibody (clone 145-2C11, BD) and free anti-mouse CD28 antibody ( $2 \mu \mathrm{g} / \mathrm{ml}$, clone $37.51, \mathrm{BD})$ for 4 days. The Treg conversion assay was described elsewhere (5). In brief, FACS-sorted splenic $\mathrm{CD}^{+} \mathrm{CD} 25^{-} \mathrm{T}$ cells were cultured with macrophages at 1:5, 1:10 and 1:20 E:T ratios in plates coated with $5 \mu \mathrm{g} / \mathrm{ml}$ anti-mouse CD3e antibody and free recombinant murine IL2 (5 ng/ml, PeproTech, Rocky Hill, NJ) in for 5 days. Control T cells were cultured with recombinant mouse TGF- $\beta 1$ ( $5 \mathrm{ng} / \mathrm{ml}$, R\&D) in cRPMI without macrophages.

In vivo experiments: For evaluation of macrophages in vivo, tumor cells were subcutaneously injected into congenic mice, such as 4T1.2 cells and EMT6 cells $\left(1 \times 10^{6}\right)$ in BALB/cJ and $\mu \mathrm{MT}$ mice, and B16-F10, and AT3 and MC-38 cells $\left(1 \times 10^{6}\right)$ in 
C57BL/6J, $\mathrm{J}_{\mathrm{H}} \mathrm{T}$ or Mb1-EYFP mice. ID8-p53--RFP cells $\left(5 \times 10^{6}\right)$ were intraperitoneally (i.p.) injected into C57BL/6J mice. For in vivo B-MF generation study, C57BL/6J mice with ID8-p53--RFP cells $\left(5 \times 10^{6}\right)$ in PeC were intraperitoneally injected with BM Lin' $\mathrm{CD} 19^{+} \mathrm{EYFP}^{+} \mathrm{B}$ cells $\left(5 \times 10^{6}\right)$ sort-purified from Mb1-cre-EYFP mice and then 7 days later, the PeC lavage cells were FACS evaluated. To evaluate tumor-supporting role of B$\mathrm{MF}$ in vivo, $\mu \mathrm{MT}$ mice were intravenously injected with $3 \times 10^{5}$ in vitro-generated B-MF or PBS 3 and 7 days after subcutaneous challenge with B16-F10 melanoma cells (day $0, n=5$ ). Tumor volume $\left(\mathrm{V}=\mathrm{a} \times \mathrm{b}, \mathrm{mm}^{2}\right)$ was measured at days $11,14,16,18$, and 21 , and at day 21 , mice were euthanized to evaluate tumor weight and $\mathrm{T}$ cells.

Cellular cholesterol content quantification: Macrophages were fixed with $4 \%$ formaldehyde solution in TBS for 5 mins, then after TBS washes, they were incubated with Filipin III at 1:100 dilution in TBS $(5 \mathrm{mg} / \mathrm{ml}$ stock in 100\% ethanol, Cayman, Ann Arbor, MI) for 60 mins in the dark. Cells were washed with TBS and lipids were quantified with Zeiss LSM 710 and Fiji software, as described above.

mRNA microarray: For the collected biological samples, the standard RNA extraction protocol was performed by RNeasy Plus Micro kits (QIAGEN, Hilden, Germany), and genome-wide expression was measured using the Agilent platform (Mouse 8X60K v2 and Hs 8 X60K v3, Agilent, Santa Clara, CA, USA) according to the manufacture's instruction. Principal Component Analysis (PCA) was performed using the Prcomp R function with expression values. Differential expressed genes (DEGs) was assessed using the moderated (empirical Bayesian) t-test implemented in the limma package (version 3.14.4)(44), and correction for multiple hypothesis testing was accomplished by calculating the Benjamini-Hochberg false discovery rate. Enriched 
pathways were discovered by GSEA tool (45) with Molecular Signature Database v7.4.All microarray analyses were performed using the $\mathrm{R}$ environment for statistical computing (version 3.6.2).

scRNA-seq: Sort purified single cell suspensions were loaded into a $10 \times$ Chromium controller (10x Genomics, Pleasanton, CA, USA) and converted to barcoded single cell RNA expression library according to the standard protocol of the Chromium Next GEM Single cell 3' kit (v3.1 chemistry) in Laboratory of Immunology and Molecular Biology, National Institute on Aging, and the single cell 3' gene expression libraries were sequenced on NovaSeq 6000 (Illumina, San Diego, CA, USA) in the Genomics Core facility of the Johns Hopkins School of Medicine. Raw sequencing data was processed using the Cell Ranger version 5.0 (10x Genomics, Pleasanton, CA, USA) pipeline. The raw gene expression matrix was normalized and scaled using the SCTransform method (40) in the Seurat R package (version 4.0) (47). The minimum number of detected genes was set to 1,000 , and genes were chosen when they were detected in more than 3 cells. Dimension reduction was performed using principal component analysis (PCA). For visualizing the generated clustering, we used the Uniform Manifold Approximation and Projections (UMAP) plot. We defined clusters with a leiden algorithm using shared nearest neighbor $(\mathrm{SNN})$ in PCA space. From in vitro B-MF and Mo-MF, we generated a total of 12 clusters for in vitro samples (0-11). Integration of in vivo samples with canonical correlation analysis (CCA) was performed, and we generated 13 clusters for in vivo tumor macrophage samples (0-12). Finally, we performed a nonparametric Wilcoxon rank-sum test to search for highly expressed genes in the clusters. In addition, human tumor single cell transcriptomes were downloaded from GEO (GSE114725, and GSE146026) and also 
processed with the same pipeline described. We used only macrophage clusters for downstream analysis. All single-cell analyses were performed using the $\mathrm{R}$ environment for statistical computing (version 4.0.5).

ATAC- seq analysis: We utilized a Hi-Seq 2000 machine to sequence the ATAC-seq libraries. We prepared 12 pair-end ATAC-seq libraries including BM CSF1R (+/-) and Spleen CSF1R (+/-) samples ( $\mathrm{n}=3$ per group). In total, 369M reads were sequenced and average $31 \mathrm{M}$ reads were sequenced per sample. We applied NIEHS TaRGETII ATAC-seq pipelines, which are available to the genomics community. All raw reads were trimmed using cutadapt package, and trimmed reads (>36bp minimum alignment length) were mapped against the mm10 reference genome using BWA aligner (48). We used deduplicated and uniquely mapped reads for peak calling analysis after excluding black-list regions defined by ENCODE (49). The candidate peaks were predicted by MACS peak calling tool (50). In addition, we also applied the DESeq2 (51) to determine differentially accessible regions (DARs); cutoff: Fold change $>1.5, \log 2 \mathrm{CPM}>1.2$, FDR $<0.05$. The differentially accessible regions were submitted for search of potential transcription factor binding sites using HOMER software (52). We used non DARs as background regions in de novo motif analysis.

Statistical analysis: The results are presented as the mean with each individual data point or in bar graph \pm SEM. GraphPad Prism (Prism 6; GraphPad Software, Inc) was used to perform statistical analysis. Data were analyzed sing Welch t-test or one-way ANOVA. A P-value less than 0.05 was considered significant $(* * * * \mathrm{P}<0.0001, * * * \mathrm{P}<0.001, * *$ $\mathrm{P}<0.01, * \mathrm{P}<0.05)$. 
ACKNOWLEDGMENTS: We are grateful to Mrs. Jane Trepel with help with human BC and OC samples; Dr. Xin Wang (NIA) for help with confocal microscopy, Drs. Elin Lehrman, Yongqing Zhang, and Supriyo De (Genomics Core, LGG, NIA) for help with performing microarray experiments and initial data analyses, and data submission to GSE; and Dr. Chandamany Arya (Lilly) for proofreading.

AUTHOR CONTRIBUTIONS: C. C. performed the research, collected and analyzed data; E. Ra., M.B. and L.Z., performed experiments; B.P. worked on bioinformatics analyses, JMM provided clinical trial samples; A.B. and I.B. supervised the study and wrote the manuscript; and A.B. conceived and designed the study.

FUNDING: This research was supported by the Intramural Research Program of the National Institute on Aging, NIH.

COMPETING INTERESTS: The authors are employees of National Institutes of Health, the U.S. government, and declare no competing interests, and there are no patents or patent applications related to this work.

DATA AND MATERIALS AVAILABILITY: The data associated with this study can be found in the paper or supplementary materials and ATAC-seq data are deposited at $\underline{\text { https://www.ncbi.nlm.nih.gov/geo/query/acc.cgi?acc }=\underline{\text { GSE178716 }} \text { and }=\text { GSE180285 }}$

SUPLEMENTAL MATERIALS: Figures S1-S8 and Tables S1-4. 


\section{REFERENCE}

1. S. N. Ganti, T. C. Albershardt, B. M. Iritani, A. Ruddell, Regulatory B cells preferentially accumulate in tumor-draining lymph nodes and promote tumor growth. Sci Rep 5, 12255 (2015).

2. C. Lee-Chang et al., Aging Converts Innate B1a Cells into Potent CD8+ T Cell Inducers. J Immunol 196, 3385-3397 (2016).

3. Y. Gu et al., Tumor-educated B cells selectively promote breast cancer lymph node metastasis by HSPA4-targeting IgG. Nat Med 25, 312-322 (2019).

4. Q. Li et al., Adoptive transfer of tumor reactive B cells confers host T-cell immunity and tumor regression. Clin Cancer Res 17, 4987-4995 (2011).

5. P. B. Olkhanud et al., Tumor-evoked regulatory B cells promote breast cancer metastasis by converting resting $\mathrm{CD}^{+} \mathrm{T}$ cells to T-regulatory cells. Cancer Res 71, 3505-3515 (2011).

6. P. B. Olkhanud et al., Thymic stromal lymphopoietin is a key mediator of breast cancer progression. Journal of immunology 186, 5656-5662 (2011).

7. K. Wejksza et al., Cancer-produced metabolites of 5-lipoxygenase induce tumorevoked regulatory B cells via peroxisome proliferator-activated receptor alpha. Journal of immunology 190, 2575-2584 (2013).

8. S. Kusmartsev, D. I. Gabrilovich, Effect of tumor-derived cytokines and growth factors on differentiation and immune suppressive features of myeloid cells in cancer. Cancer Metastasis Rev 25, 323-331 (2006).

9. K. Movahedi et al., Different tumor microenvironments contain functionally distinct subsets of macrophages derived from Ly6C(high) monocytes. Cancer Res 70, 5728-5739 (2010).

10. E. Ragonnaud et al., Tumor-Derived Thymic Stromal Lymphopoietin Expands Bone Marrow B-cell Precursors in Circulation to Support Metastasis. Cancer Res 79, 5826-5838 (2019).

11. M. Lelekakis et al., A novel orthotopic model of breast cancer metastasis to bone. Clin.Exp.Metastasis 17, 163-170 (1999).

12. M. Bodogai et al., Immunosuppressive and Prometastatic Functions of MyeloidDerived Suppressive Cells Rely upon Education from Tumor-Associated B Cells. Cancer Res 75, 3456-3465 (2015).

13. P. B. Olkhanud et al., Breast cancer lung metastasis requires expression of chemokine receptor CCR4 and regulatory T cells. Cancer Res. 69, 5996-6004 (2009).

14. H. Xie, M. Ye, R. Feng, T. Graf, Stepwise reprogramming of B cells into macrophages. Cell 117, 663-676 (2004).

15. S. L. Nutt, B. Heavey, A. G. Rolink, M. Busslinger, Commitment to the Blymphoid lineage depends on the transcription factor Pax5. Nature 401, 556-562 (1999).

16. T. Audzevich et al., Pre/pro-B cells generate macrophage populations during homeostasis and inflammation. Proc Natl Acad Sci U S A 114, E3954-E3963 (2017). 
17. E. R. Stanley, M. Cifone, P. M. Heard, V. Defendi, Factors regulating macrophage production and growth: identity of colony-stimulating factor and macrophage growth factor. J Exp Med 143, 631-647 (1976).

18. S. R. Almeida et al., Mouse B-1 cell-derived mononuclear phagocyte, a novel cellular component of acute non-specific inflammatory exudate. Int Immunol 13, 1193-1201 (2001).

19. D. Kitamura, J. Roes, R. Kuhn, K. Rajewsky, A B cell-deficient mouse by targeted disruption of the membrane exon of the immunoglobulin mu chain gene. Nature 350, 423-426 (1991).

20. J. Chen et al., Immunoglobulin gene rearrangement in B cell deficient mice generated by targeted deletion of the JH locus. Int Immunol 5, 647-656 (1993).

21. E. Hobeika et al., Testing gene function early in the B cell lineage in mb1-cre mice. Proc Natl Acad Sci U S A 103, 13789-13794 (2006).

22. W. Chen, P. Ten Dijke, Immunoregulation by members of the TGFbeta superfamily. Nat Rev Immunol 16, 723-740 (2016).

23. L. M. Francisco, P. T. Sage, A. H. Sharpe, The PD-1 pathway in tolerance and autoimmunity. Immunol Rev 236, 219-242 (2010).

24. G. Gimpl, Cholesterol-protein interaction: methods and cholesterol reporter molecules. Subcell Biochem 51, 1-45 (2010).

25. H. Ohno et al., A c-fms tyrosine kinase inhibitor, Ki20227, suppresses osteoclast differentiation and osteolytic bone destruction in a bone metastasis model. Mol Cancer Ther 5, 2634-2643 (2006).

26. A. Delogu et al., Gene repression by Pax 5 in B cells is essential for blood cell homeostasis and is reversed in plasma cells. Immunity 24, 269-281 (2006).

27. A. P. Ng et al., An Erg-driven transcriptional program controls B cell lymphopoiesis. Nat Commun 11, 3013 (2020).

28. S. Taoudi et al., ERG dependence distinguishes developmental control of hematopoietic stem cell maintenance from hematopoietic specification. Genes Dev 25, 251-262 (2011).

29. S. R. Himes, S. Cronau, C. Mulford, D. A. Hume, The Runx1 transcription factor controls CSF-1-dependent and -independent growth and survival of macrophages. Oncogene 24, 5278-5286 (2005).

30. M. R. Corces et al., Lineage-specific and single-cell chromatin accessibility charts human hematopoiesis and leukemia evolution. Nat Genet 48, 1193-1203 (2016).

31. E. Azizi et al., Single-Cell Map of Diverse Immune Phenotypes in the Breast Tumor Microenvironment. Cell 174, 1293-1308 e1236 (2018).

32. B. Izar et al., A single-cell landscape of high-grade serous ovarian cancer. Nat Med 26, 1271-1279 (2020).

33. T. A. Wynn, A. Chawla, J. W. Pollard, Macrophage biology in development, homeostasis and disease. Nature 496, 445-455 (2013).

34. M. Donadon et al., Macrophage morphology correlates with single-cell diversity and prognosis in colorectal liver metastasis. J Exp Med 217, (2020).

35. H. Jeon, S. C. Blacklow, Structure and physiologic function of the low-density lipoprotein receptor. Annu Rev Biochem 74, 535-562 (2005).

36. J. H. Madenspacher et al., Cholesterol 25-hydroxylase promotes efferocytosis and resolution of lung inflammation. JCI Insight 5, (2020). 
37. C. L. Chen, S. S. Huang, J. S. Huang, Cholesterol modulates cellular TGF-beta responsiveness by altering TGF-beta binding to TGF-beta receptors. J Cell Physiol 215, 223-233 (2008).

38. F. Shojaei et al., G-CSF-initiated myeloid cell mobilization and angiogenesis mediate tumor refractoriness to anti-VEGF therapy in mouse models. Proc Natl Acad Sci U S A 106, 6742-6747 (2009).

39. Z. Qin et al., B cells inhibit induction of T cell-dependent tumor immunity. Nature Medicine 4, 627-630 (1998).

40. S. Gaidamakov et al., Targeted deletion of the gene encoding the La autoantigen (Sjogren's syndrome antigen B) in B cells or the frontal brain causes extensive tissue loss. Mol Cell Biol 34, 123-131 (2014).

41. P. H. Hand, P. F. Robbins, M. L. Salgaller, D. J. Poole, J. Schlom, Evaluation of human carcinoembryonic-antigen (CEA)-transduced and non-transduced murine tumors as potential targets for anti-CEA therapies. Cancer Immunol Immunother 36, 65-75 (1993).

42. M. E. Gatti-Mays et al., A Phase II Single Arm Pilot Study of the CHK1 Inhibitor Prexasertib (LY2606368) in BRCA Wild-Type, Advanced Triple-Negative Breast Cancer. Oncologist 25, 1013-e1824 (2020).

43. J. M. Lee et al., Prexasertib, a cell cycle checkpoint kinase 1 and 2 inhibitor, in BRCA wild-type recurrent high-grade serous ovarian cancer: a first-in-class proof-of-concept phase 2 study. Lancet Oncol 19, 207-215 (2018).

44. M. E. Ritchie et al., limma powers differential expression analyses for RNAsequencing and microarray studies. Nucleic Acids Res 43, e47 (2015).

45. A. Subramanian et al., Gene set enrichment analysis: a knowledge-based approach for interpreting genome-wide expression profiles. Proc Natl Acad Sci U $S$ A 102, 15545-15550 (2005).

46. C. Hafemeister, R. Satija, Normalization and variance stabilization of single-cell RNA-seq data using regularized negative binomial regression. Genome Biol 20, 296 (2019).

47. Y. Hao et al., Integrated analysis of multimodal single-cell data. Cell 184, 35733587 e3529 (2021).

48. H. Li, R. Durbin, Fast and accurate long-read alignment with Burrows-Wheeler transform. Bioinformatics 26, 589-595 (2010).

49. H. M. Amemiya, A. Kundaje, A. P. Boyle, The ENCODE Blacklist: Identification of Problematic Regions of the Genome. Sci Rep 9, 9354 (2019).

50. Y. Zhang et al., Model-based analysis of ChIP-Seq (MACS). Genome Biol 9, R137 (2008).

51. M. I. Love, W. Huber, S. Anders, Moderated estimation of fold change and dispersion for RNA-seq data with DESeq2. Genome Biol 15, 550 (2014).

52. S. Heinz et al., Simple combinations of lineage-determining transcription factors prime cis-regulatory elements required for macrophage and B cell identities. Mol Cell 38, 576-589 (2010). 
Figure 1. Cancer induces differentiation of macrophages from BM B cells. A and B, FACS staining frequency \pm SEM of co-expression for markers of B cells (CD19) and macrophages $\left(\mathrm{F} 4 / 80^{+}\right)$in $\mathbf{A}, \mathrm{BALB} / \mathrm{CJ}$ and $\mu \mathrm{MT}$ mice with orthotopic $4 \mathrm{~T} 1.2$ breast cancer $(\mathrm{n}=5-6)$ and $\mathbf{B}, \mathrm{C} 57 \mathrm{BL} / 6$ and $\mathrm{J}_{\mathrm{H}} \mathrm{T}$ mice with spontaneous ovarian cancer (Mogp-tag and Mogp- $\mathrm{J}_{\mathrm{H}} \mathrm{T}$, respectively, $\mathrm{n}=4$ ) Frequency of $\mathrm{F} 4 / 80^{+} \mathrm{CD} 11 \mathrm{~b}^{+}$cells within $\mathrm{CD} 19^{+} \mathrm{TIBs}$ (left panels) and $\mathrm{CD}_{19}{ }^{+} \mathrm{IgM}^{+}$cells within $\mathrm{F} 4 / 80^{+}$TAM (right panels). C, Representative flow plot for $\mathrm{F} 4 / 80^{+} \mathrm{CD} 11 \mathrm{~b}^{+}$macrophage of $\mathrm{B}$ cells isolated from $\mathrm{BM}$, spleen, $\mathrm{LN}$ and $\mathrm{PeC}$ treated with CM from 4T1.2 breast cancer cells. D, Representative Giemsa staining in vitro-generated B-MF. Scale bar represented $20 \mu \mathrm{m}$. E, Representative FACs plots of $\mathrm{F} 4 / 80^{+} \mathrm{CD} 11 \mathrm{~b}^{+}$macrophage of BM B-cells treated with CM from 4T1.2, EMT6, AT3, B16F10, and MC-38 cells. F and G Representative FACs plots for CD68 and CD11b staining of pre-B cell line (70z/3)_after 30-day stimulation with (RPMI) or without 4T1.2-CM and composite frequency analysis of $\mathrm{F} 4 / 80^{+} \mathrm{CD} 11 \mathrm{~b}^{+}$and $\mathrm{CD} 68^{+} \mathrm{CD} 11 \mathrm{~b}^{+}$cells from four independent experiments. H, Representative Imagestream imaging for surface markers F4/80, CD11b, CD19, and CD20 on in vitro-generated B-MF from naïve Mb1-cre-EYFP ${ }^{+}$ mice (bottom two panels, H) and WT mice (top two panels, H). I, Frequency \pm SEM of $\mathrm{CD} 274^{+}$and $\mathrm{LAP}^{+}$cells within $\mathrm{EYFP}^{+}$or $\mathrm{EYFP}^{+} \mathrm{F} 4 / 80^{+} \mathrm{CD} 11 \mathrm{~b}^{+}$cells quantified 7-day after i.p. injection of BM B cells from naïve Mb1-cre-EYFP mice in C57BL/6 mice with ID8 ovarian cancer in $\mathrm{PeC}(\mathrm{n}=3,) . * * * * \mathrm{P}<0.0001, * * * \mathrm{P}<0.001, * * \mathrm{P}<0.01, * \mathrm{P}<0.05$ between indicated comparisons.

Figure 2. Distinct gene expression profiles of B-MF and Mo-MF. A, Representative FACS plots of $\mathrm{F} 4 / 80^{+} \mathrm{CD} 11 \mathrm{~b}^{+}$macrophage of $\mathrm{BM} \mathrm{B}$ cells or monocytes post 7-day treatment with 4T1.2-CM. Macrophage phenotype gated as $\mathrm{F} 4 / 80^{+} \mathrm{CD} 11 \mathrm{~B}^{+}$from $\mathrm{B}$ cell 
(B-MF) and monocytes (Mo-MF) B, Representative expression of B-cell markers CD79a and IgM by FACs analysis on B-MF (Red) and Mo-MF (Orange). C, PCA plot of mRNA expression profiles generated from microarray data of sort purified B-MF (Blue), Mo-MF (Orange) and BM B cells (Green) (n=3). D and E, Bar plots of GSEA predicted pathways enriched in B-MF (D) or Mo-MF cells (E) from the Molecular Signature Database. F and G, UMAP plot of B-MFs (10,563 cells) and Mo-MFs (10,235 cells) analyzed using Seurat with colors depicting clusters by cell type $(\mathbf{F})$ or by gene expression $(\mathbf{G})$. H, Heatmap of top genes with differential expression characterizing the 12 major cell types of in vitro BMF and Mo-MF single cell clusters. I, Overlay of Mrc1 expression for in vitro B-MF and Mo-MF single cells. J, UMAP plot of sort purified TAMs (10,885 cells) generating 13 unique cell clusters from tumor tissues of 4 mice with $4 \mathrm{~T} 1.2$ cancers. $\mathbf{K}$, Violin plots of the expression of three in vitro derived B-MF up-regulated DEGs (Egr1, Ier3 and Slc40a1) in in vivo mouse 4T1.2 TAM single cell clusters.

Figure 3. B-MF and Mo-MF are functionally different. A, Ki67 staining (upper) and BrdU uptake (lower panel) frequency of B-MF and Mo-MF (frequency \pm SEM, n=2). B and C, Representative Filipin III staining images and quantification (MFI). D and E, Representative images and quantifications of $\mathrm{RFP}^{+}$apoptotic cells in B-MF and Mo-MF cultured with apoptotic ID8-RFP ovarian cancer cells for 2 h. Eight representative fields per sample were quantified and scale bars represent $20 \mu \mathrm{m}(\mathrm{C}$ and E). F, Representative histograms of proliferated $\mathrm{CD}^{+} \mathrm{T}$ cells after co-culturing with macrophages at 1:10 (E:T) ratio in the presence of anti-CD3/CD28 Abs for 4 days. Control T cells were cultured alone with (activated) or without (non-activated) anti-CD3/CD28 Abs. Numbers represent 
percentage of proliferated T cells. Macrophages were generated from BM B-cell precursors (B-MF) and monocytes (Mo-MF) with 4T1.2-CM treatment. The results in B - F were independently reproduced at least three times. $\mathbf{G}-\mathbf{J}$, B-MF support tumor growth in vivo. $\mu \mathrm{MT}$ mice were i.v. transferred with $3 \times 10^{5}$ in vitro-generated B-MF or PBS 3 and 7 days after s.c. challenge with B16-F10 melanoma cells $(n=5)$. Each symbol is for a single mouse; $\mathrm{Y}$-axis is for $\% \pm \mathrm{SEM}$ of tumor-infiltrated $\mathrm{CD}^{+} \mathrm{T}$ cells in $\mathrm{CD}^{+} 5^{+}$cells $(\mathbf{H})$ and IFN $\gamma^{+}$in $\mathrm{CD}^{+} \mathrm{T}$ cells (I) and tumor weight $\pm \operatorname{SEM}(\mathbf{J}) . * * * * \mathrm{P}<0.0001, * * * \mathrm{P}<0.001, * *$ $\mathrm{P}<0.01,{ }^{*} \mathrm{P}<0.05$ between indicated comparisons.

Figure 4. CSF1/CSF1R signaling axis is critical for B-MF differentiation. A, Numbers of $\mathrm{CD} 19^{+}$(left) and $\mathrm{CD} 93^{+} \mathrm{CD} 19^{+}$(middle) B cells and frequency of $\mathrm{CSF} 1 \mathrm{R}^{+}$within $\mathrm{CD} 93^{+} \mathrm{CD} 19^{+} \mathrm{B}$ cells (right) in the spleen of naïve and 4T1.2 tumor-bearing mice $(\mathrm{n}=5)$. B, ELISA measurements of secreted M-CSF in media conditioned by 4T1.2, EMT6, AT3, MC-38, B16-F10, and ID8 cells $(\mathrm{n}=6, \mathrm{pg} / \mathrm{ml})$. C, Representative FACS plots of the $\mathrm{F} 4 / 80^{+} \mathrm{CD} 11 \mathrm{~b}^{+}$B-MF converted from sort-purified $\mathrm{BM} \mathrm{CSF} 1 \mathrm{R}^{+}$and $\mathrm{CSF} 1 \mathrm{R}^{-}$B-cell precursors and splenic CSF1R ${ }^{+}$and CSF1R- B cells after 7-day treatment with 4T1.2-CM. D and E, Representative FACS plots of $\mathrm{F} 4 / 80^{+} \mathrm{CD} 11 \mathrm{~b}^{+}(\mathrm{B}-\mathrm{MF})$ from BM B-cell precursors after 7-day treatment with 4T1.2-CM (D) and pre-B cell line 70z/3 after 30-day treatment with 4T1.2-CM (E) with and without anti-MCSF Ab or Ki-20227 treatments (a specific MCSF-R inhibitor). F and $\mathbf{G}$, Representative FACS plot (F) and quantification $(\mathbf{G})$ of the generation of B-MF after 7-day 4T1.2-CM treatment on cells isolated from WT or mice with conditional CSF1R deficiency in B cells (Mb1-CSF1R Flox/Flox $^{\text {( }}(\mathrm{n}=6)$. Results for all panels were independently reproduced minimally three times. $* * * * \quad \mathrm{P}<0.0001$, *** $\mathrm{P}<0.001, * * \mathrm{P}<0.01, * \mathrm{P}<0.05$ between indicated comparisons. 
Figure 5. Cancer targets BM CSF1R ${ }^{+} \mathbf{P A X 5}^{\text {Low }}$ B-cell precursors. A, FACs analysis of Pax5 expression of freshly isolated BM CSF1R ${ }^{-}$or $\mathrm{CSF}_{1 \mathrm{R}^{+}} \mathrm{B}$ cell precursors $(\mathrm{n}=5,) \mathbf{B}$, FACs analysis of Pax 5 expression of pre-B cell (70z/3 cells) treated with indicated cancer CM (n=3). C, FACs analysis of Pax5 expression of BM Lin-CD19+B220+CD93+IgMIgD-CSF1R+ B cell precursors treated with M-CSF for 48h $(n=3)$. D, 3D PCA plot of chromatin accessibility generated from ATAC-seq data of BM CSF1R ${ }^{+}$and CSF1R- B-cell precursors and spleen $\mathrm{CSF}_{1 \mathrm{R}^{+}}$and CSF1R- follicular B cells $\mathbf{E}$, Heatmap of differentially

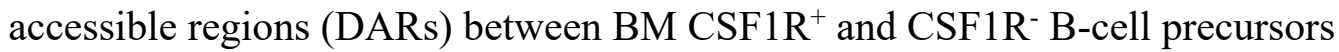
$(\mathrm{FDR}<0.05)$. F, Top, highly significant de novo motifs are more open in BM CSF1R ${ }^{+}$ compared to BM CSF1R ${ }^{-}$as well as Spleen $\mathrm{CSF} \mathrm{R}^{+}$and $\mathrm{CSF}^{-} \mathrm{R}^{-}$cells (678 sites). $\mathbf{G}$, mRNA microarray heatmap of macrophage related-DEGs in B cells isolated from PB of patients with breast cancer $(\mathrm{BC}, \mathrm{n}=8)$ compared to healthy donors $(\mathrm{HD}, \mathrm{n}=7)$. Scale bar is for expression z-score. H, Cell frequency of CSF1R ${ }^{+}$(left), $\mathrm{CD}^{+} 8^{+}$(middle) and LDLR ${ }^{+}$ (right) cells within $\mathrm{CD} 19^{+} \mathrm{CD} 10^{+} \mathrm{B}$ cells of $\mathrm{PB}$ from healthy donors (HD) or patients with ovarian cancer (OC) ( $\mathrm{n}=5-7)$. I and $\mathbf{J}$, UMAP of scRNA sequencing data of macrophage cells (left) and expression levels of in vitro B-MF enriched genes (right) in published human BC (I) and OC (J) datasets. Error bars represent SEM, **** $\mathrm{P}<0.0001$, $* * * \mathrm{P}<0.001, * * \mathrm{P}<0.01, * \mathrm{P}<0.05$ between indicated comparisons. 

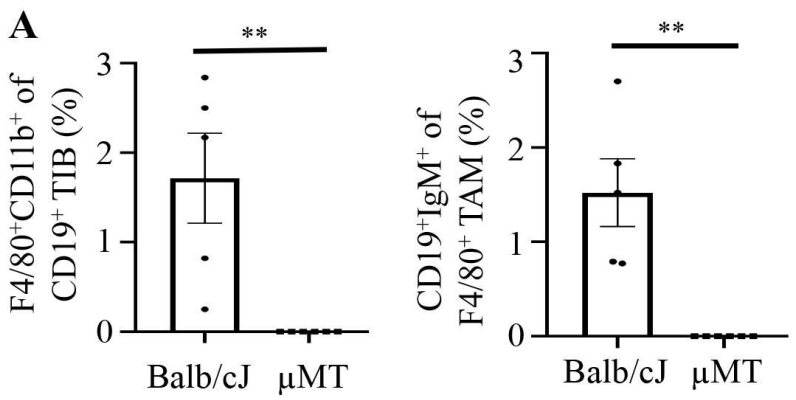

B
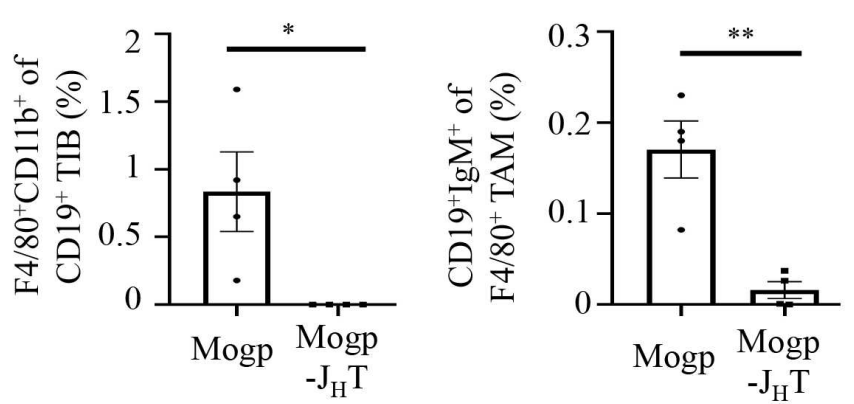

C

BM

Spleen

LN

D

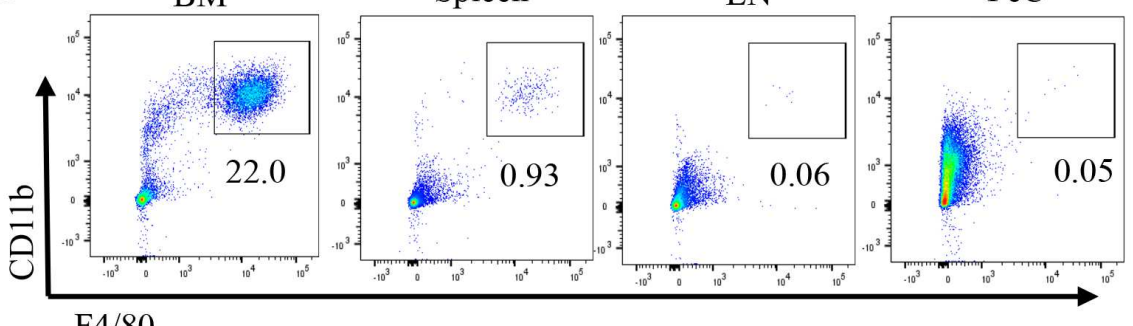

E

F4/80

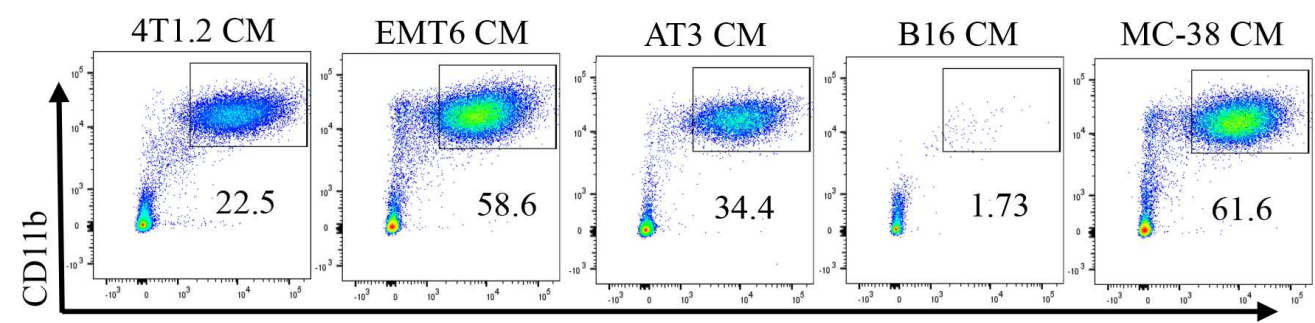

F4/80

F
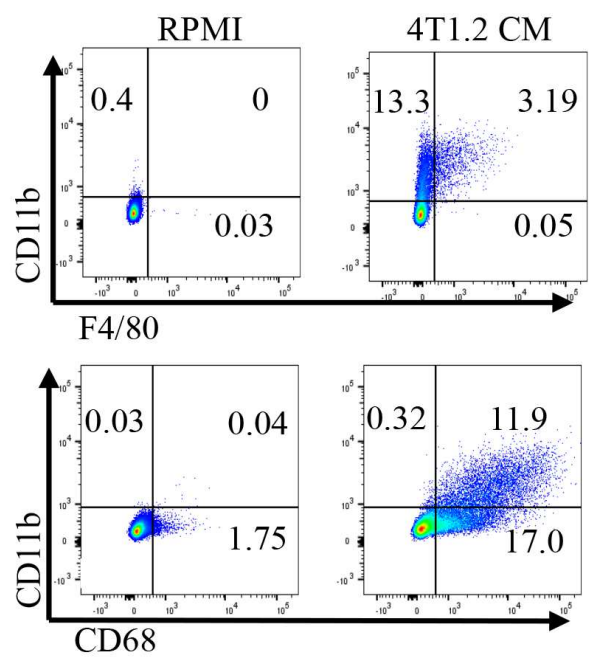

G
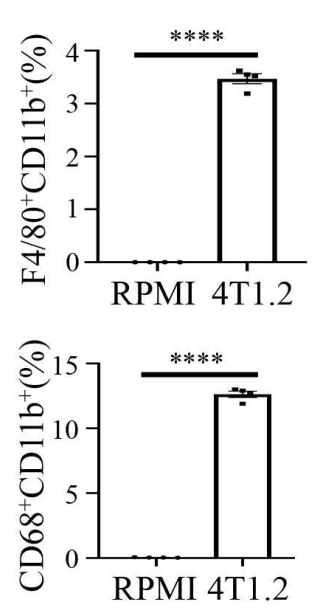

H

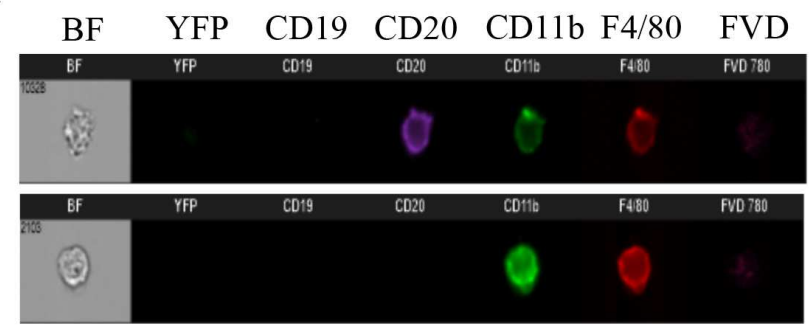

Mb1-cre-

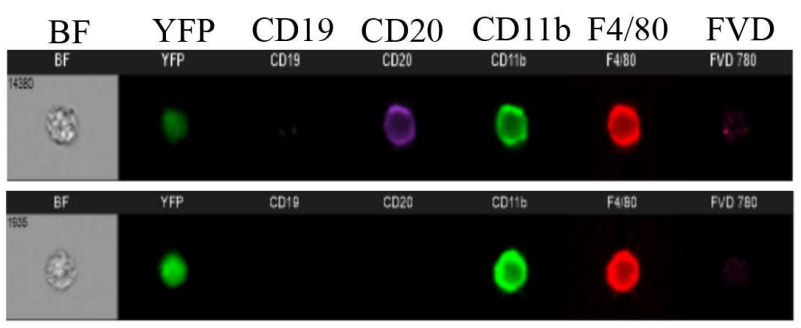

I
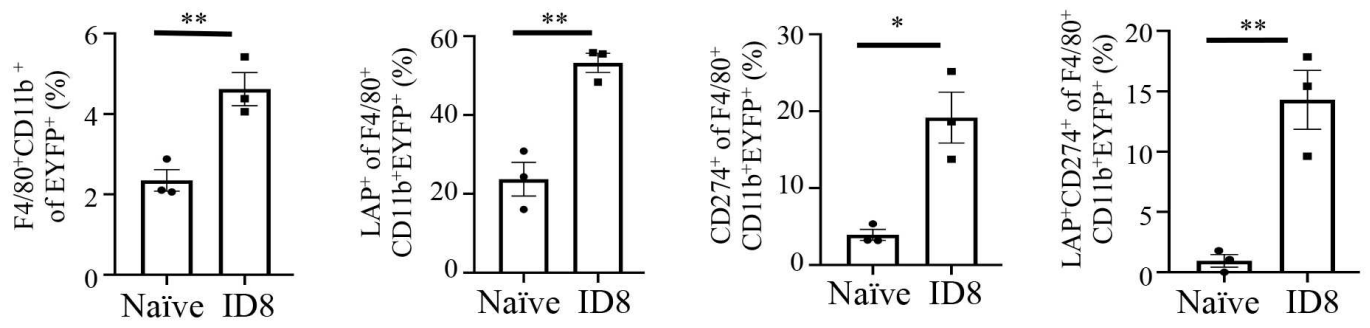

Figure 1 


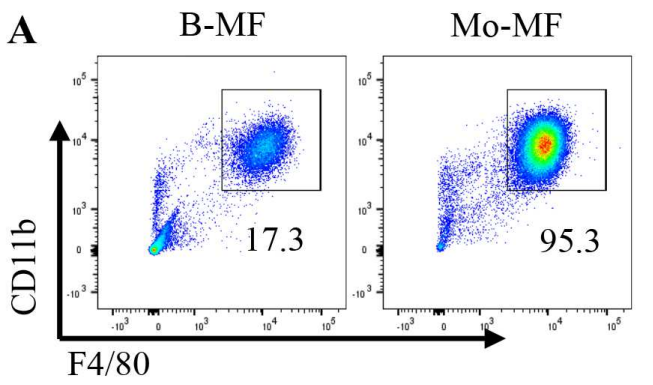

D

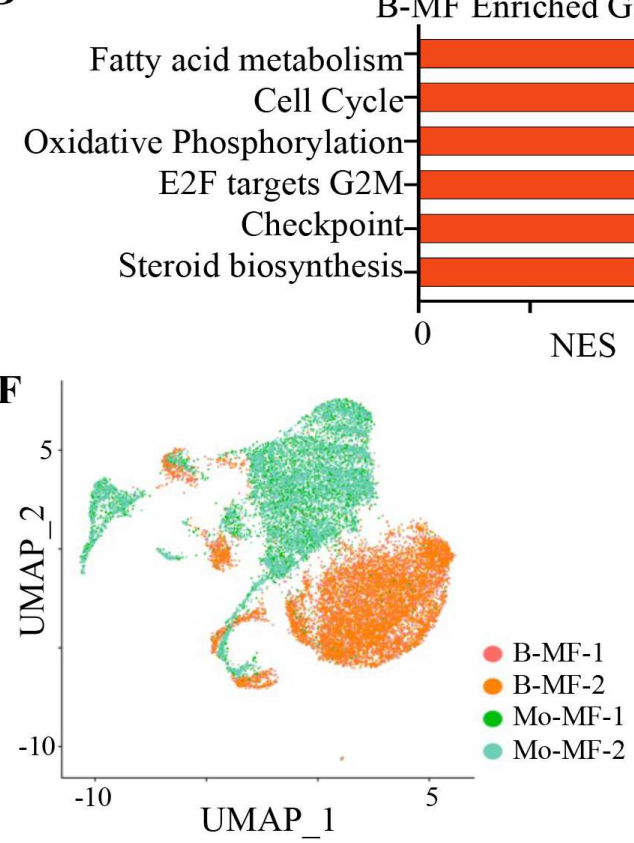

B-MF Enriched Gene sets

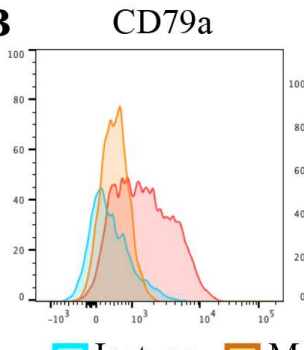

$\square$ Isotype $\square$ Mo-MF $\square$ B-MF

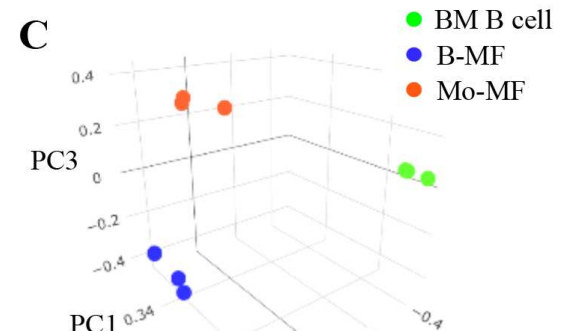

E

$0.3^{3}-0, \mathrm{PC} 2$

Mo-MF Enriched Gene sets

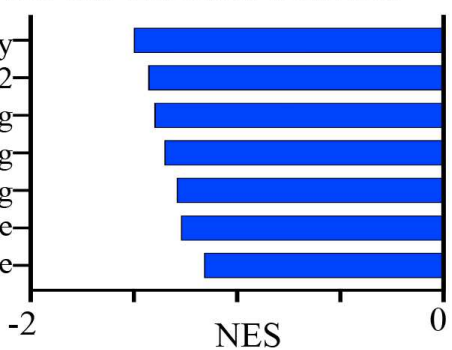
Interferon gamma response M1 up-regulation vs M2 KRAS signaling IL2 STAT5 signalingTGF beta signalingInflammatory response$+1.5$
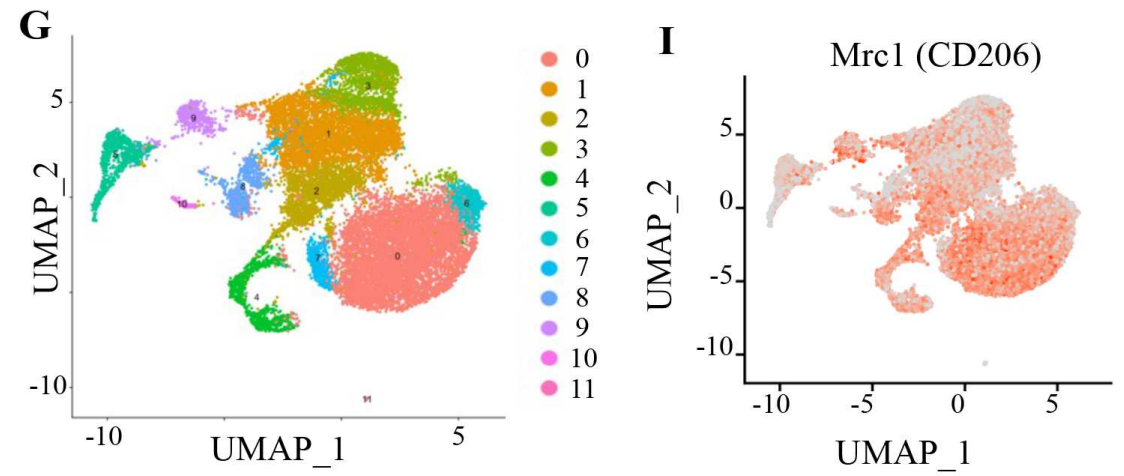
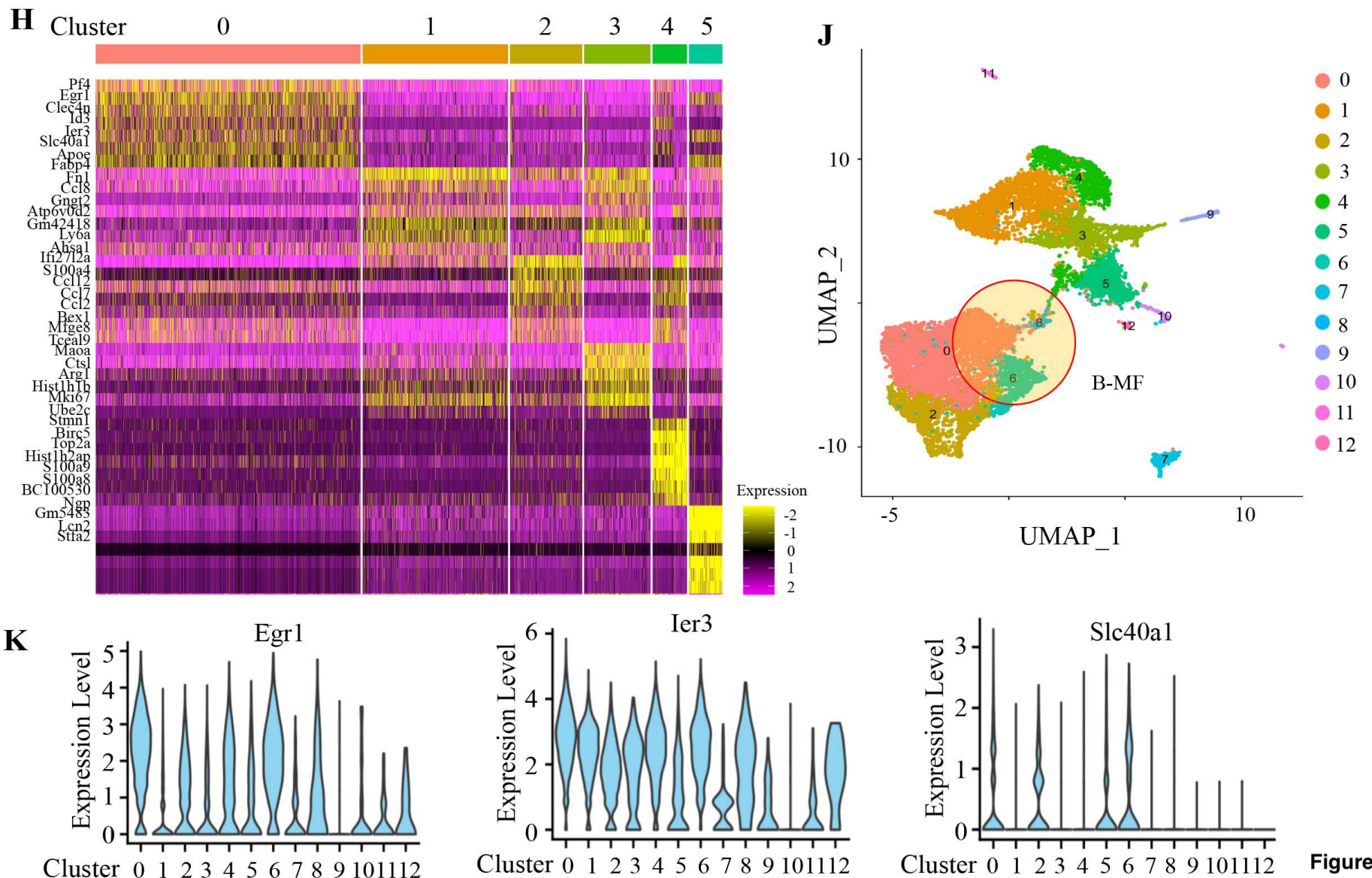

Cluster 01223456789101112

Figure 2 
A

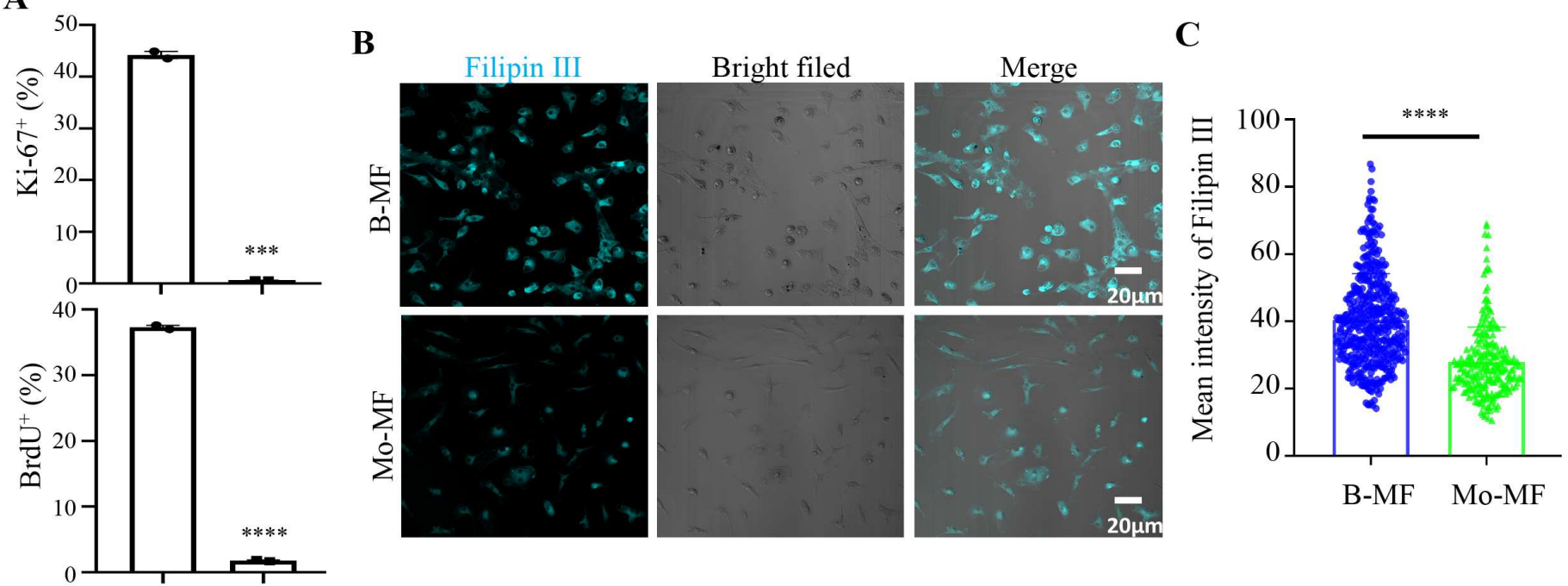

D
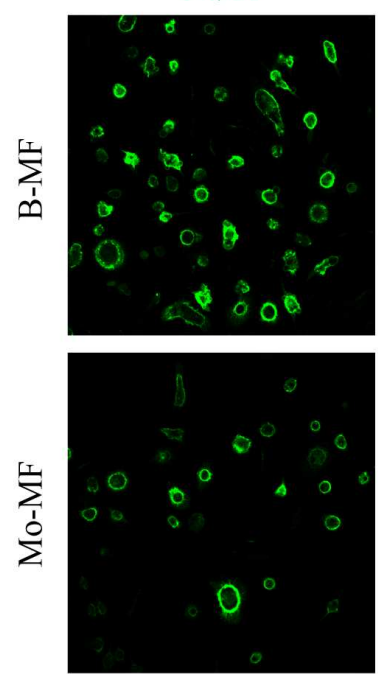

RFP
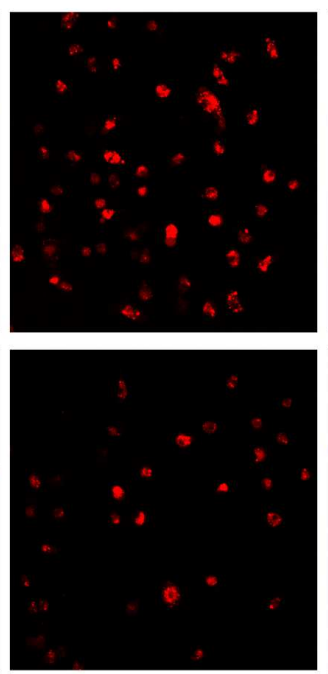

DAPI
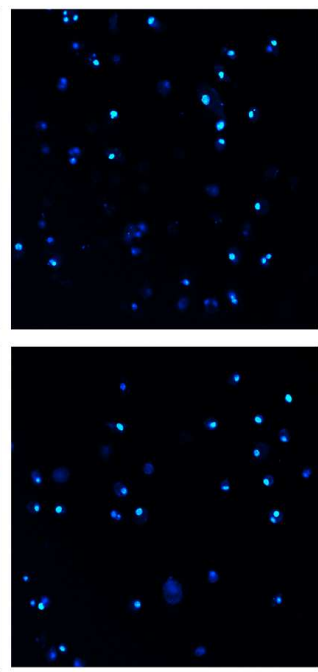

Merge
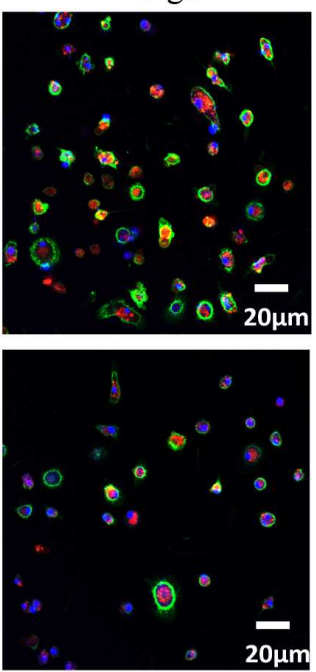

$\mathbf{E}$
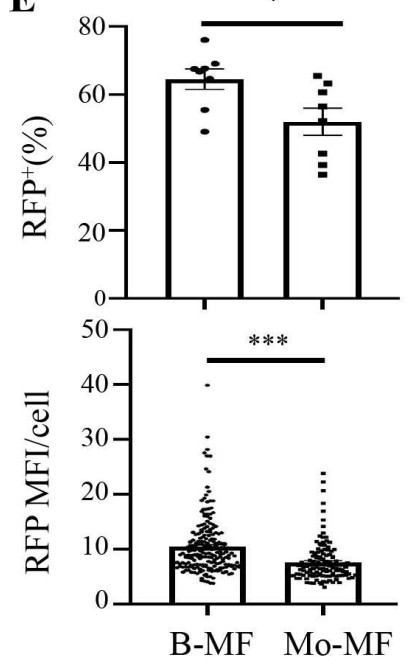

F

Unactivated

Activated

B-MF:T=1:10

Mo-MF:T=1:10

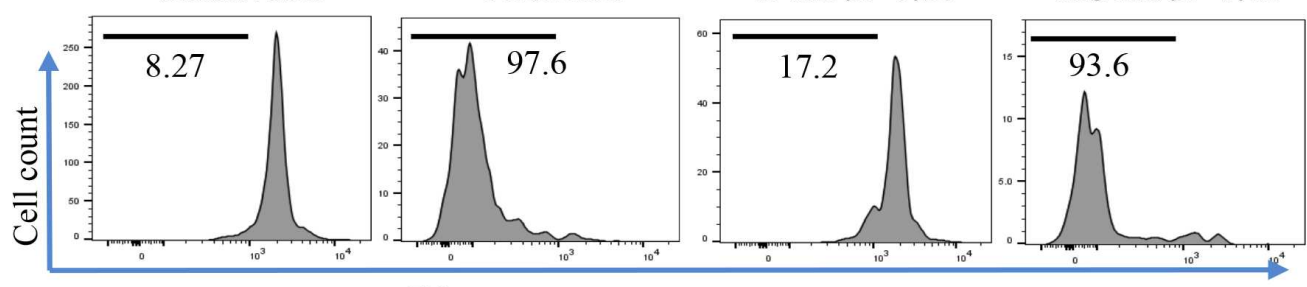

Proliferation dye eFluor ${ }^{\mathrm{TM}} 450 \mathrm{CD} 4^{+} \mathrm{T}$ cells

G

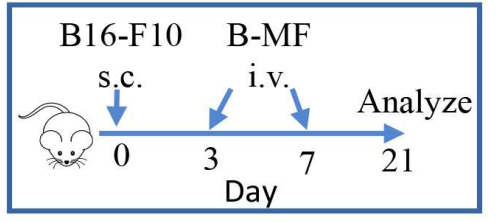

Figure 3
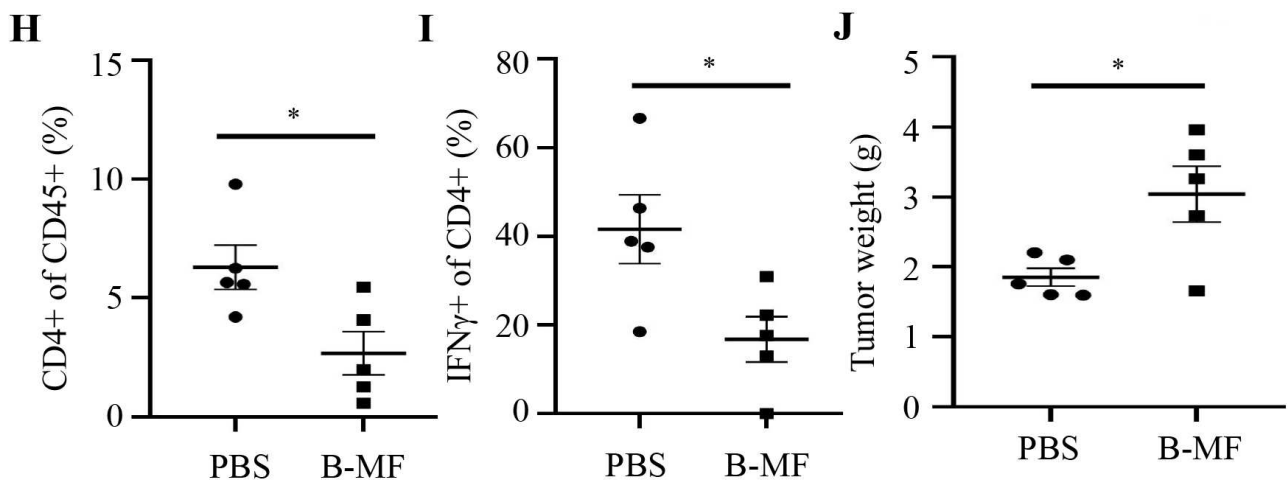
A

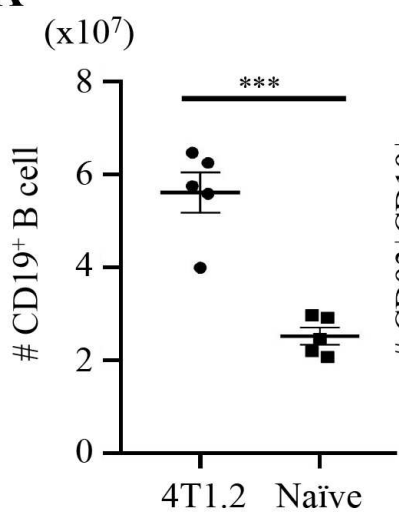

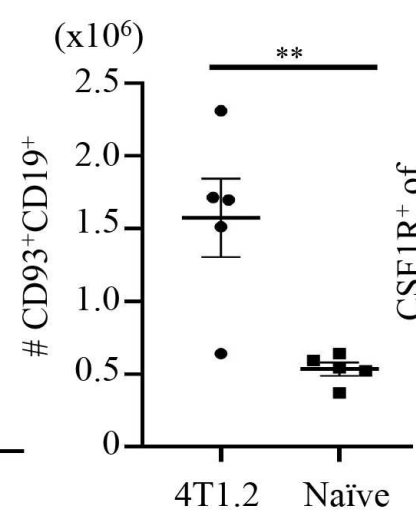

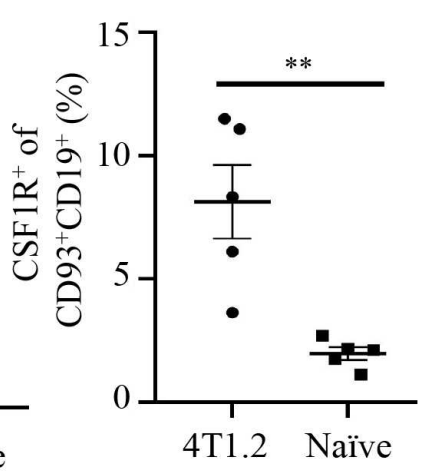

B
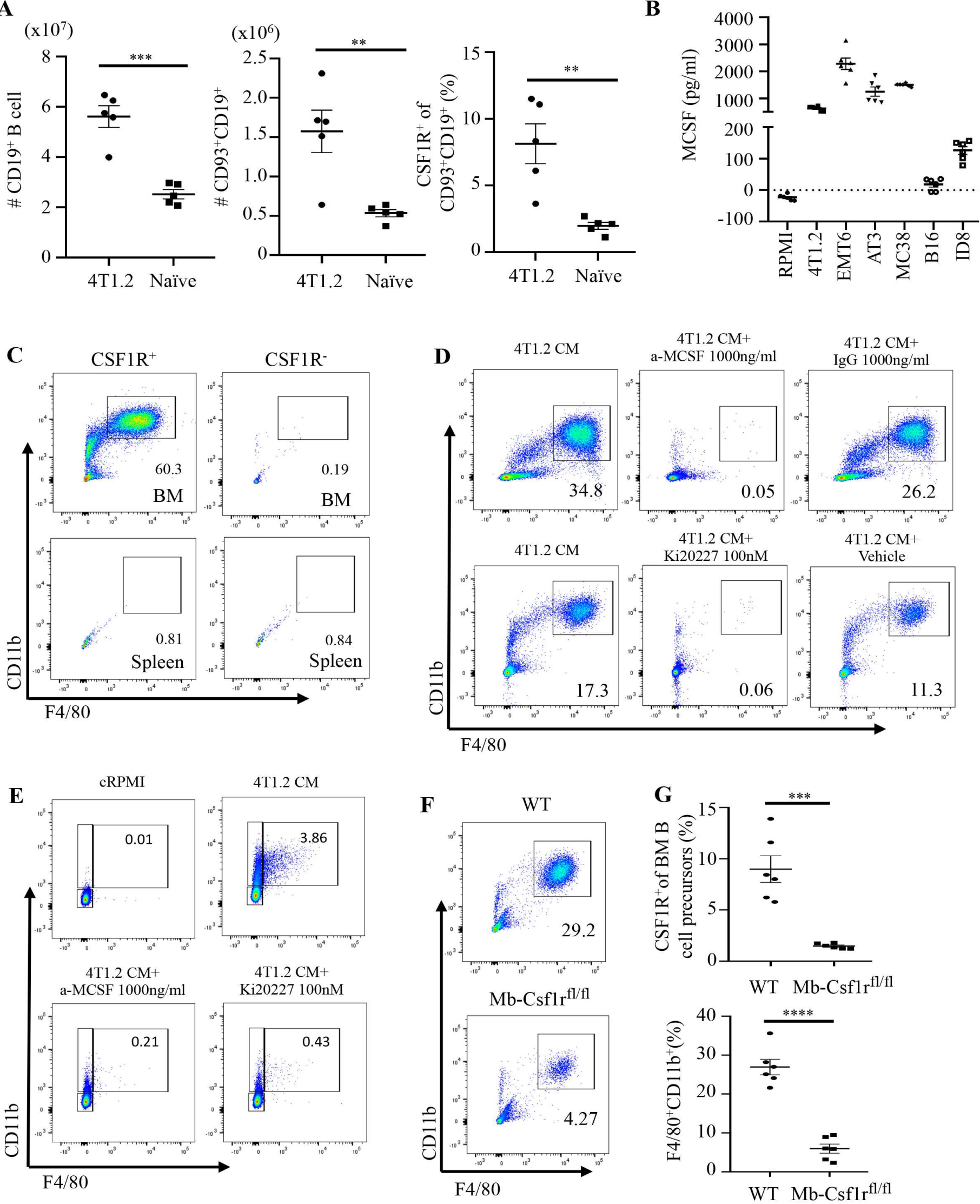
A

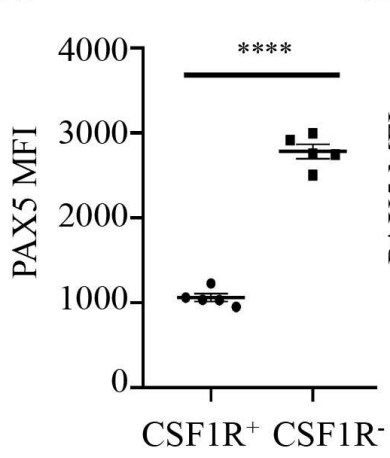

B

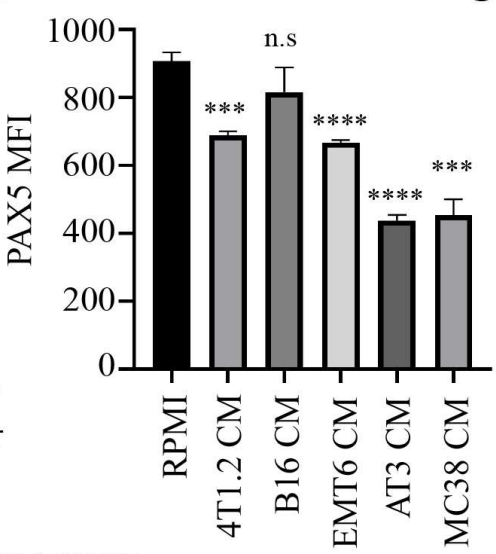

C

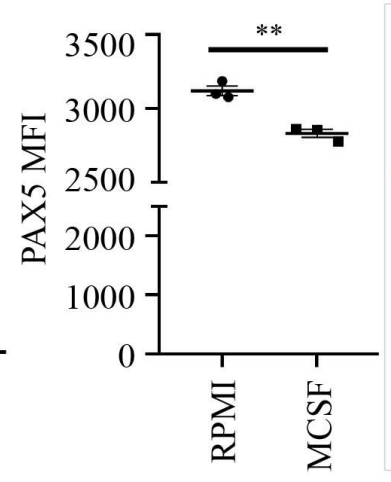

D
ATAC-seq (PCA)

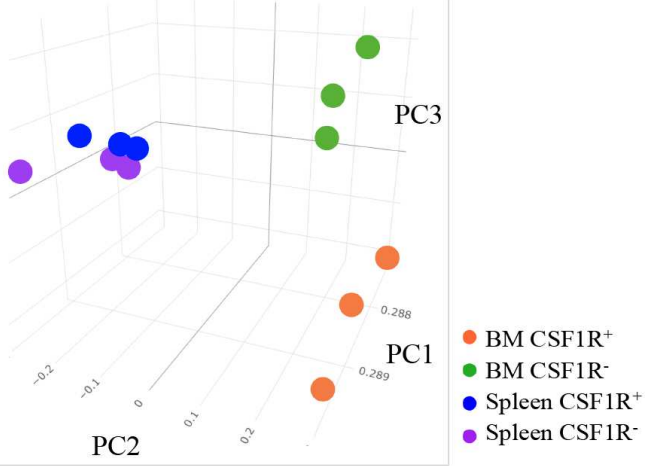

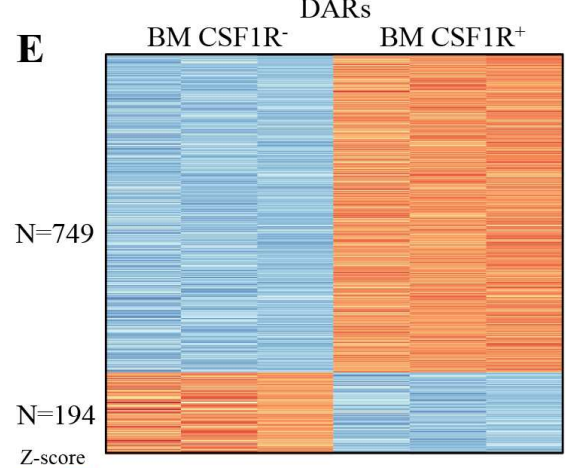

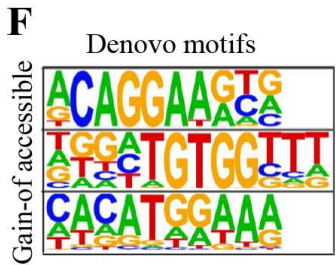

ERG (1e-35)

RUNX1 (1e-34)

NFAT5 (1e-12)

678 sites

$\left(\mathrm{BM} \mathrm{CSF} 1 \mathrm{R}^{+}>\right.$Spleen \& BM CSF1R-)

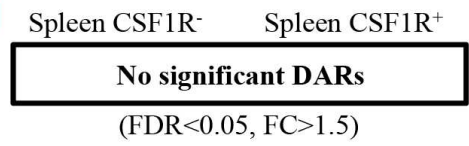

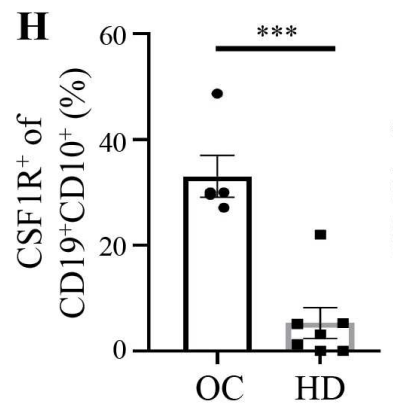
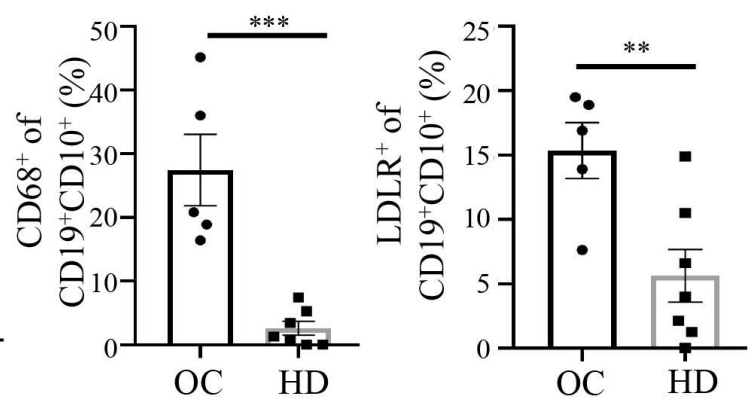

G

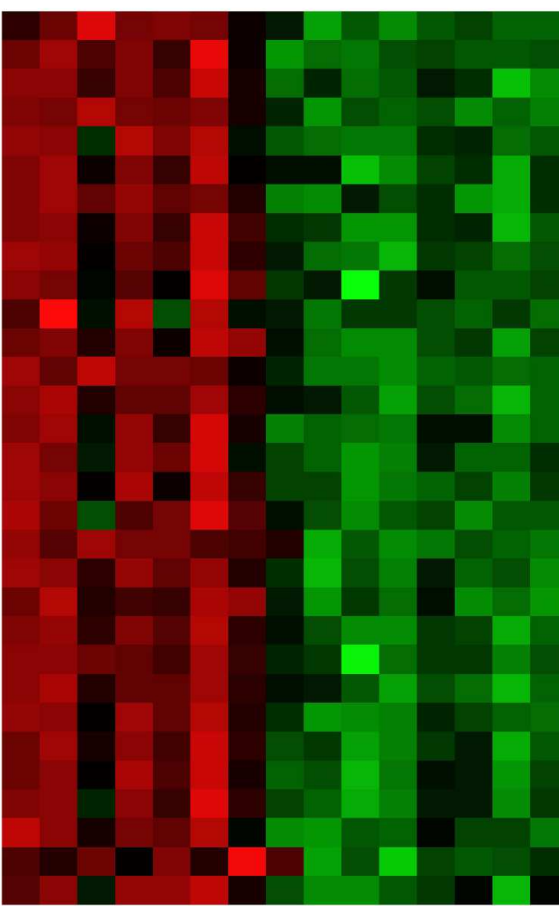

SLC40A1 CREG1 CYFIP1 MFSD1 HMOX1 NINJ1 VASH1 CD163 FCGR1B P2RX7 CCL3 LILRB4 MPP1 CCR1 CEBPA MS4A7 FAM20C MARCO ITGB3 CX3CR1 CCR2 CD14 CD36 CCR1 CSF1R CSF3R CD33 CD302 CCR5 LAMP5 ITGAX

Breast cancer Healthy donor $\begin{array}{lll}-2 & 0 & 2\end{array}$ Row z-score
I

Breast cancer clusters

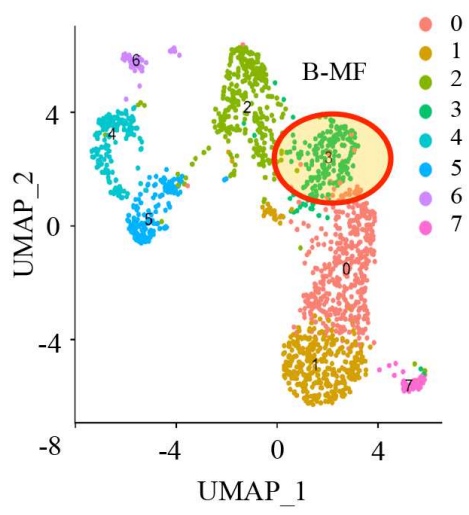

B-MF enriched genes

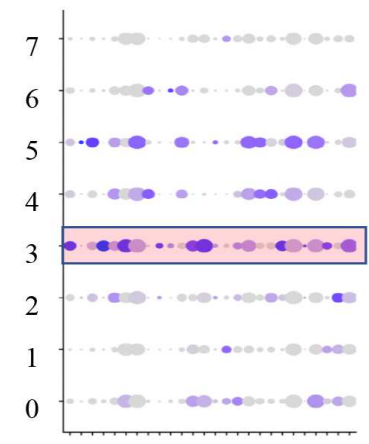

Genes (sorted p-value)

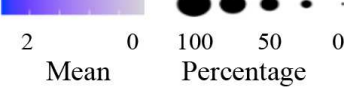

J Ovarian cancer clusters

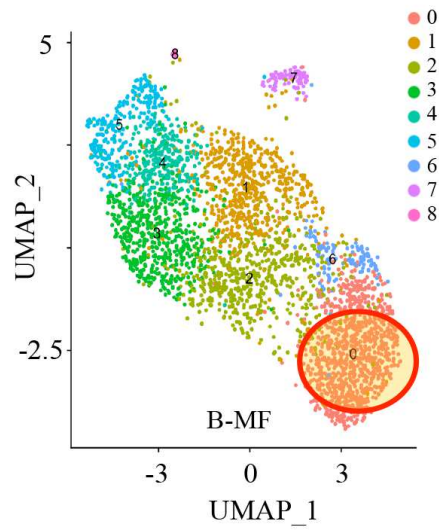

B-MF enriched genes

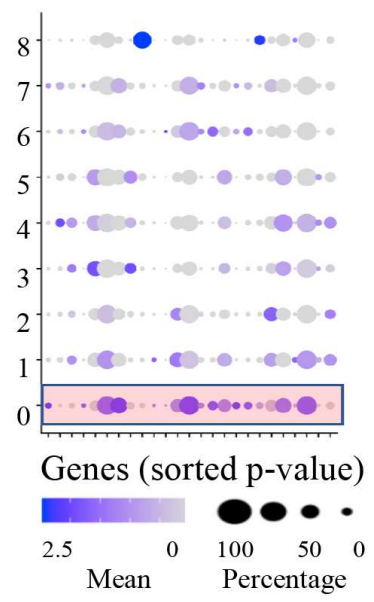

Figure 5 


\section{Supplementary Files}

This is a list of supplementary files associated with this preprint. Click to download.

- SupplementaryTables14.xlsx

- SuppIFigsSI.pdf 